\title{
GENERALIZED GROUP PRESENTATION AND FORMAL DEFORMATIONS OF CW COMPLEXES
}

\author{
RICHARD A. BROWN
}

\begin{abstract}
A Peiffer-Whitehead word system $\mathscr{W}$, or generalized group presentation, consists of generators, relators (words of order 2), and words of higher order $n$ that represent elements of a free crossed module $(n=3)$ or a free module $(n>3)$. The $P_{n}$-equivalence relation on word systems generalizes the extended Nielsen equivalence relation on ordinary group presentations. Word systems, called homotopy readings, can be associated with any connected $\mathrm{CW}$ complex $K$ by removing a maximal tree and selecting one generator or word per cell, via relative homotopy. Given homotopy readings $\mathscr{W}_{1}$ and $\mathscr{W}_{2}$ of finite $\mathrm{CW}$ complexes $K_{1}$ and $K_{2}$ respectively, we show that $\mathscr{W}_{1}$ is $P_{n}$-equivalent to $\mathscr{W}_{2}$ if and only if $K_{1}$ formally $(n+1)$-deforms to $K_{2}$. This extends results of P. Wright (1975) and W. Metzler (1982) for the case $n=2$. For $n \geq 3$, it follows that $\mathscr{W}_{1}$ is $P_{n}$-equivalent to $\mathscr{W}_{2}$ if and only if $K_{1}$ and $K_{2}$ have the same simple homotopy type.
\end{abstract}

1.1 Introduction. The purpose of this paper is to reintroduce J. H. C. Whitehead's homotopy systems from the viewpoint of R. Peiffer. In [8], extended group presentations (" $\Sigma$-systems") are considered that have three fields, namely generators, relators and identities among relators ("relation identities"). At the same time, Whitehead [14] introduced the homotopy system of a CW complex $K$, consisting of the fundamental group $\rho_{1}=\pi_{1}\left(K^{1}\right)$ and the relative homotopy groups $\rho_{n}=\pi_{n}\left(K^{n}, K^{n-1}\right) \quad(n>1)$ with the standard boundary maps $d_{n}: \rho_{n} \rightarrow \rho_{n-1}$. We generalize Peiffer's approach using homotopy systems as a guide, by defining generalized group presentations, or word systems, with an arbitrary number of component fields. Each entry or word in such a field gives rise to an element in the free module $\rho_{n}$, much as a relator represents an element of a free group in an ordinary group presentation.

After setting up some machinery, we prove the following generalization of P. Wright's Theorem [15].

Received by the editors June 8, 1987.

1980 Mathematics Subject Classification (1985 Revision). Primary 57M05, 20F05; Secondary 57Q10; 57Q05.

Key words and phrases. Extended Nielsen transformations, simple homotopy equivalence, relative homotopy groups; homotopy systems, $\Sigma$-systems; characteristic maps, upper semicontinuous decomposition; transiency. 
Theorem (1.1.1). (See (6.1.1).) Two $C W$ complexes $K, L$ of dimension at most $n$ formally $(n+1)$-deform to each other, $K_{\wedge}^{n+1} L$, iff any corresponding word systems $\mathscr{W}(K)$ and $\mathscr{W}(L)$ are $P_{n}$-equivalent, as defined in Subsection 2.3.

Here, $K^{n+1} \curvearrowright L$ means that there is a sequence of elementary expansions and collapses of dimension at most $n+1$ that transforms $K$ to $L$, and $P_{n}$ equivalence is a generalization of the equivalence relation introduced in [1].

The proof of this theorem in the more difficult case $\left(\stackrel{n+1}{\wedge} \Rightarrow P_{n}\right)$ ultimately rests on an analysis of the structure of words in a word system. In fact, if $e$ is an $n$-cell in an intermediate complex $K^{\prime}$ that is collapsed during the deformation $K^{\prime} \wedge L$, then the "spelling" of the word corresponding to $e$ in $\mathscr{W}\left(K^{\prime}\right)$ may be used as a prescription for converting the collapse of $e$ into a $P_{n}$-equivalence of word systems. See Theorem (4.1.2).

In view of [12], we may conclude that simple homotopy equivalence of $\mathrm{CW}$ complexes of dimension $n \geq 3$ is characterized by $P_{n}$-equivalence of corresponding word systems. For $n=2$, this statement would be equivalent to the Andrews-Curtis conjecture [1]. It is possible that an analysis of $P_{3}$-equivalence of word systems of order 2 would shed some light on that conjecture.

Word systems offer a flexible means to express geometric deformations in an algebraic manner. For example, in order to manage the effect of geometric 3-deformations on corresponding group presentations, Wright transformed a given 3-deformation of 2-complexes into a transient deformation in which at most one 3-cell is present at any time. In contrast, the proof of Theorem (1.1.1) permits an accumulation of $(n+1)$-cells in an $(n+1)$-deformation: the words in a word system express the information needed to carry out the proof.

Theorem (1.1.1) can be used to show that Wright's transiency property holds in higher dimensions. See Proposition (7.1.2).

This work requires a technical result that may be of interest, concerning 3dimensional cells in a CW complex. If $e_{1}^{2}, \ldots, e_{n}^{2}$ are free faces of a 3 -cell $e^{3}$, then there exists a characteristic map $\phi$ of $e^{3}$ that "realizes the freeness" of all of those faces simultaneously, i.e., $\phi$ restricts to a characteristic map for each 2-cell $e_{i}^{2}$ (see Proposition (3.1.2)). This fills an apparent gap in S. Young's proof of Wright's Theorem for the CW category [16]. Our proof requires a theorem of Dyer and Hamstrom [6].

This paper is organized as follows. The remainder of $\S 1$ reviews preliminary material. We define the notion of a word system or generalized group presentation in $\S 2$. The correspondence between CW complexes and word systems is discussed. Various equivalence relations patterned after EN- and Q-moves (see Subsection 1.3) are proposed for word systems. In $\S 3$, several technical results are established. In $\S \S 4$ and 5, certain of the equivalence relations defined in $\S 2$ are shown to have identical equivalence classes, which proves most of Theorem (1.1.1). The proof of that theorem is completed in $\S 6$. Section 7 contains applications.

I am indebted to Professor Craggs, who directed this dissertation and suggested the problem of proving Wright's Theorem using $\Sigma$-systems. I am grateful to the referee for his comments, which were helpful, and for suggesting the transiency problem addressed by Proposition (7.1.2). 
1.2 Notation and conventions. For elements $a$ and $b$ in a topological space $X$, a path $p: a \rightsquigarrow b$ from $a$ to $b$ is a continuous function $p: I \rightarrow X$ that satisfies $p(0)=a, p(1)=b$.

We are interested in finite, connected $\mathrm{CW}$ complexes $K$. The $m$-skeleton $K^{m}$ of $K$ is the subcomplex consisting of all cells in $K$ of dimension at most $m$. Let $B^{m}$ denote a closed topological $m$-ball, and suppose that $\mu$ is the index of an $m$-cell in $K$. Unless other indicated, $e^{m}$ will always be understood to be an m-cell in a CW complex, $\phi^{m}: B^{m} \rightarrow K$ will be a characteristic map for $e^{m}=$ $\phi^{m}$ (int $\left.B\right)$, and $f^{m}$ will be the attaching map $f^{m}=\partial \phi^{m}$ : bdy $B^{m} \rightarrow K^{m-1}$. The same relationships will hold for decorated symbols, e.g., $\bar{e}_{\mu}^{m}, \bar{\phi}_{\mu}^{m}, \bar{f}_{\mu}^{m}$.

Proposition (1.2.1). Suppose that $\phi, \psi: B^{m} \rightarrow K^{m}$ are two characteristic maps for the same $m$-cell $e^{m} \in K$ of a $C W$ complex $K$ that agree on the basepoint * of $B^{m}, \phi(*)=\psi(*)=*_{K}$.

Then the relative homotopy classes $[\phi]$ and $[\psi]$ in $\pi_{m}\left(K^{m}, K^{m-1}, *_{K}\right)$ agree up to orientation reversal and the action of $\pi_{1}\left(K^{m-1}, *_{K}\right)$, that is,

$$
[\phi]=\alpha \cdot[\psi]^{\varepsilon} \text { for some } \alpha \in \pi_{1}\left(K^{m-1}, *_{K}\right), \varepsilon= \pm 1 .
$$

Proof. This is easily proved using [13, V(1.4)] and the Relative Hurewicz Theorem.

Given two maps $f_{0}, f_{1}:\left(X, A, *_{X}\right) \rightarrow(Y, B, B)$ and a path $p: f_{0}\left(*_{X}\right) \leadsto$ $f_{1}\left(*_{X}\right)$ in $B$, we say that $f_{0}$ is freely homotopic to $f_{1}$ via the path $p, f_{0} \simeq_{p} f_{1}$, if there is a homotopy $H: X \times I \rightarrow Y$ satisfying $H\left|X \times 0=f_{0}, H\right| X \times 1=f_{1}$, $H \mid A \times I \subset B$, and $H \mid *_{X} \times I=p$. The symbol $\simeq$ denotes based homotopy, i.e., free homotopy via the constant path. The notation $[f]$ will generally be used to denote the (absolute or relative) based homotopy class of a map.

A topological ball tuple $\left(B^{m}, B_{1}^{m-1}, \ldots, B_{Q}^{m-1}\right)$ is a closed $m$-ball together with $Q$ disjoint closed $(m-1)$-balls $B_{1}^{m-1}, \ldots, B_{Q}^{m-1}$ that are contained in bdy $\left(B^{m}\right)$. Suppose that $e^{m}, e_{1}^{m-1}, \ldots, e_{Q}^{m-1}$ are cells in a CW complex $K$. We say that a characteristic map $\phi^{m}: B^{m} \rightarrow K$ is a multiple collapse map for $\left(e^{m}, e_{1}^{m-1}, \ldots, e_{Q}^{m-1}\right)$ if there is a ball tuple structure $\left(B^{m}, B_{1}^{m-1}, \ldots, B_{Q}^{m-1}\right)$ on $B^{m}$ such that

a. $\phi^{m} \mid B_{q}^{m-1}$ is a characteristic map for the $(m-1)$-cell $e_{q}^{m-1}$, and

b. $\phi^{m} \mid\left(B^{m}-B_{q}^{m-1}\right)$ does not meet $e_{q}^{m-1}$.

In the case $Q=1$, we call $\phi^{m}$ a (single) collapse map.

Let $K$ and $L$ be $\mathrm{CW}$ complexes satisfying $L=K \cup e^{m-1} \cup e^{m}$, and suppose that a collapse map $\phi^{m}$ exists for $\left(e^{m}, e^{m-1}\right)$. The move $[K, L]$ is an elementary m-expansion, written $K \underset{e, m}{\nearrow} L$. The move $[L, K]$ is an elementary m-collapse, $L \underset{e, m}{\searrow} K$. An elementary $m$-deformation is an elementary $m$-expansion or $m$-collapse. An elementary $m$-deformation is said to have $d i$ mension $m$.

A formal $m$-deformation, $K \wedge$, $L$, is a finite sequence of complexes $K=$ $K_{0}, K_{1}, \ldots, K_{Q}=L$ such that each move $\left[K_{q}, K_{q+1}\right]$ is an elementary deformation of dimension at most $m$. That formal $m$-deformation is transient if $K$ and $L$ are $(m-1)$-complexes and every $m$-cell is collapsed immediately after it is expanded. When no deformation $\left[K_{q}, K_{q+1}\right]$ has dimension less than $n$, 
we write $K \widehat{N}_{n}^{m} L$. If all those moves are elementary expansions or collapses, we write $K \nearrow^{m} L$ or $K \searrow_{m}^{m} L$.

Definition (1.2.2). A move $[K, L]$ is an elementary homotopy $m$-move, $K \underset{e, m}{\rightarrow}$ $L$, if there are cells $e^{m} \in K$ and $\bar{e}^{m} \in L$ such that

(a) $K=K^{\prime} \cup e^{m}$ and $L=K^{\prime} \cup \bar{e}^{m}$ for some common subcomplex $K^{\prime}$, and

(b) those cells have attaching maps $f^{m}$ and $\bar{f}^{m}$ that are freely homotopic in $K^{\prime}$.

Such a homotopy move is said to have dimension $m$. If the image of the homotopy in condition (b) is contained in a subcomplex $K_{0} \subset K^{\prime}$, we say that the homotopy move takes place within $K_{0}$. By the Cellular Approximation Theorem, we may adjust any homotopy satisfying condition (b) to insure that the homotopy move takes place within the complex $\left(K_{0}\right)^{m}$.

A sequence $K=K_{1}, K_{2}, \ldots, K_{Q}=L$ is a homotopy m-deformation, $K \stackrel{m}{\sim} L$, if each move $\left[K_{q}, K_{q+1}\right]$ is an elementary expansion, collapse or homotopy move, of dimension at most $m$.

Suppose that $K \underset{e, m}{\rightarrow} L=K \cup \bar{e}^{m}-e^{m}$ is an elementary homotopy $m$-move that takes place within $K^{m}-e^{m}$. Then one may attach an $(m+1)$-cell $e^{m+1}$ to $K^{m} \cup \bar{e}^{m}$ via the attaching map

$$
f^{m+1}=h \cup\left(\phi^{m} \times 0\right) \cup\left(\bar{\phi}^{m} \times 1\right): \operatorname{bdy}\left(\mathbf{I}^{m+1}\right) \rightarrow K^{m} \cup \bar{e}^{m}
$$

where $h: \operatorname{bdy}\left(\mathbf{I}^{m}\right) \times \mathbf{I} \rightarrow K^{m}-e^{m}$ is the given free homotopy between the attaching maps. The $(m+1)$-expansion $K \nearrow_{e, m+1} K \cup \bar{e}^{m} \cup e^{m+1}$ is called the expansion associated with the homotopy move $[K, L]$. Likewise, $K \cup \bar{e}^{m} \cup$ $e^{m+1} \searrow_{e, m+1}\left(K-e^{m}\right) \cup \bar{e}^{m}$ is called the collapse associated with $[K, L]$. We also refer to that homotopy move as sliding the $m$-cell $e^{m}$ across the $(m+1)$-cell $e^{m+1}$ or sliding the attaching map $f^{m}$ across the homotopy $h$.

Lemma (1.2.3). Let $K$ and $L$ be $C W$-complexes. If a homotopy $n$-deformation $K \stackrel{n}{\curvearrowright} L$ exists, then there is a transient formal $(n+1)$-deformation $K^{n+1} \wedge L$. Proof. Replace each homotopy $m$-move $K_{q} \underset{e, m}{\rightarrow} K_{q+1}$ in the given homotopy $n$-deformation by the associated expansion and collapse

$$
K_{q} \underset{e, m+1}{\nearrow} K \cup \bar{e}^{m} \cup e^{m+1} \searrow_{e, m+1}\left(K-e^{m}\right) \cup \bar{e}^{m} .
$$

1.3 Extended Nielson transformations and Wright's Theorem. We now briefly review group presentations and 2-dimensional complexes in order to motivate higher-dimensional definitions and relate our results to the literature.

A finite group presentation $\mathscr{P}=\left\langle y_{1}, \ldots, y_{J} \mid R_{1}, \ldots, R_{K}\right\rangle$ consists of generators $y_{1}, \ldots, y_{J}$, which are distinct symbols, and relators $R_{1}, \ldots, R_{K}$, which are words in the symbols $y_{j}$ and their inverses. The group presented by $\mathscr{P}$ is

$$
G(\mathscr{P})=F\left(y_{1}, \ldots, y_{J}\right) / \text { n.cl. }\left(\left\{R_{1}, \ldots, R_{K}\right\}\right) .
$$

Two words are equivalent, $w_{1} \sim w_{2}$, if they represent the same element $\left\langle w_{1}\right\rangle=$ $\left\langle w_{2}\right\rangle \in G(\mathscr{P})$. Two words are congruent, $w_{1} \equiv w_{2}$, if they are identical as 
sequences of symbols. The presentations $\mathscr{P}=\left\langle y_{1}, \ldots, y_{J} \mid R_{1}, \ldots, R_{K}\right\rangle$ and $\mathscr{P}^{\prime}=\left\langle y_{1}^{\prime}, \ldots, y_{M}^{\prime} \mid R_{1}^{\prime}, \ldots, R_{N}^{\prime}\right\rangle$ are congruent if they have the same cardinalities $J=M, K=N$ and if each relator $R_{k}^{\prime}$ arises from the corresponding relator $R_{k}$ by substitution, $R_{k}^{\prime} \equiv R_{k}\left(y_{1}^{\prime}, \ldots, y_{J}^{\prime}\right)$. Words or presentations are considered to be the same only if they are congruent.

There are two common approaches to defining extended Nielsen transformations on group presentations. The moves for each approach are listed below.

EN-moves [8, 15]. Let $\mathscr{P}=\left\langle y_{1}, \ldots, y_{M} \mid R_{1}, \ldots, R_{N}\right\rangle$ be a group presentation.

EN1. Change of representing word. For some $n$, replace $R_{n}$ by a word $\bar{R}_{n}$ that represents the same element in the free group $F\left(y_{1}, \ldots, y_{M}\right)$.

EN2. Inversion of a relator. For some $n$, replace $R_{n}$ by the inverted word $\left(R_{n}\right)^{-1}$.

EN3. Product of relators. For some $n$ and $j(n \neq j)$, replace $R_{n}$ by $R_{n} R_{j}$.

EN4. Interchange of relators. For some $n$ and $j(n \neq j)$, replace $R_{n}$ by $R_{j}$ and $R_{j}$ by $R_{n}$.

EN5. Conjugation of relators. For some $m$ and $n$, replace $R_{n}$ by $y_{m} R_{n} y_{m}^{-1}$.

EN6. Expansion. Append a new generator $y_{M+1}$ and a new relator $R_{N+1} \equiv$ $y_{M+1} R$ for some word $R=R\left(y_{1}, \ldots, y_{M}\right)$ in the other generators.

EN7. Collapse. Remove $y_{M}$ and $R_{N}$. This move is permitted only if $R_{N} \equiv$ $y_{M} R$ for a word $R=R\left(y_{1}, \ldots, y_{M-1}\right)$ in the other generators, and if $y_{M}$ does not occur in $R_{1}, \ldots, R_{N-1}$.

Q-moves [9, 7]. Let $\mathscr{P}=\left\langle y_{1}, \ldots, y_{M} \mid R_{1}, \ldots, R_{N}\right\rangle$ be a group presentation. $\mathrm{Q} 1=\mathrm{EN} 1, \mathrm{Q} 2=\mathrm{EN} 2, \mathrm{Q} 3=\mathrm{EN} 3, \mathrm{Q} 4=\mathrm{EN} 5$.

Q5. Automorphism of underlying free group. For an automorphism $\sigma$ of the free group $F\left(y_{1}, \ldots, y_{M}\right)$, replace the word $R_{n}\left(y_{1}, \ldots, y_{M}\right)$ by the word $R_{n}\left(\sigma y_{1}, \ldots, \sigma y_{M}\right)$ for all $n$.

Q6. Stabilization. Append a new generator $y_{M+1}$ and a new relator $R_{N+1} \equiv$ $y_{M+1}$.

Q7. Destabilization. Remove $y_{M}$ and $R_{N}$. This move is permitted only if $R_{N} \equiv y_{M}$ and $y_{M}$ does not occur in $R_{1}, \ldots, R_{N-1}$.

Two group presentations are said to be EN-equivalent if one can be obtained from the other by a sequence of moves of the types EN1,.., EN7. Two group presentations are Q-equivalent if they are related by a sequence of moves of types $\mathrm{Q} 1, \ldots, \mathrm{Q} 4$; they are $\mathrm{Q}^{*}$-equivalent if they are related by a sequence of $\mathrm{Q} 1, \ldots, \mathrm{Q} 5$ moves; and they are $\mathrm{Q}^{* *}$-equivalent if they are related by a sequence of $\mathrm{Q} 1, \ldots, \mathrm{Q} 7$ moves.

Proposition (1.3.1) (see [4, Appendix and 7]). Two group presentations are ENequivalent if and only if they are $Q^{* *}$-equivalent.

Suppose that $K$ is a CW complex of dimension two. A group presentation is a cellular presentation for $\pi_{1}(K)$ if its generators correspond to a basis of the free group $\pi_{1}\left(K^{1}, *_{K}\right)$ and its relators are words representing the elements induced by attaching maps of the 2-cells in $K$. More precisely, each generator $y_{m}$ is associated with the homotopy class $\left[\lambda_{m}\right] \in \pi_{1}\left(K^{1}, *_{K}\right)$ of a loop $\lambda_{m}:(\mathbf{I}, \operatorname{bdy}(\mathbf{I})) \rightarrow\left(K^{1}, *_{K}\right)$. Each relator $R_{n}=R_{n}\left(y_{1}, \ldots, y_{M}\right)$ represents a homotopy class $\left[\xi_{n} \# f_{n}^{2} \# \overline{\xi_{n}}\right] \in \pi_{1}\left(K^{1}, *_{K}\right)$, where $f_{n}^{2}: \operatorname{bdy}\left(\mathbf{I}^{2}\right) \rightarrow K^{1}$ is an 
attaching map for the 2-cell $e_{n}^{2}, \xi_{n}: *_{K} \rightsquigarrow f_{n}^{2}(0)$ is a connecting path to the basepoint of $e_{n}^{2}$, \# denotes path multiplication and $\overline{\xi_{n}}$ denotes the inverse path $\overline{\xi_{n}}(t)=\xi_{n}(1-t) \quad(0 \leq t \leq 1)$.

The Seifert-Van Kampen Theorem implies that a cellular presentation for $K$ does in fact present the group $\pi_{1}\left(K, *_{K}\right)$. By a standard construction, every group presentation is a cellular presentation for some 2-complex $K(\mathscr{P})$.

Proposition (1.3.2). Suppose that $\mathscr{P}=\left\langle y_{1}, \ldots, y_{M} \mid R_{1}, \ldots, R_{N}\right\rangle$ and $\mathscr{P}^{\prime}=$ $\left\langle y_{1}^{\prime}, \ldots, y_{M}^{\prime} \mid R_{1}^{\prime}, \ldots, R_{N}^{\prime}\right\rangle$ are cellular presentations for the same 2-complex $K$.

Then $\mathscr{P}$ and $\mathscr{P}^{\prime}$ are $Q^{*}$-equivalent.

Proof. Straightforward.

The following is a consequence of our Theorem (6.1.1).

Theorem (1.3.3) (Wright's Theorem). Suppose that $K$ and $K^{\prime}$ are finite, connected $C W$ 2-complexes. Let $\mathscr{P}$ be a cellular presentation of $K$ and $\mathscr{P} \prime a$ cellular presentation of $K^{\prime}$.

Then $K$ formally 3 -deforms to $K^{\prime}, K{ }^{3} K^{\prime}$ if and only if $\mathscr{P}$ is EN-equivalent to $\mathscr{P}^{\prime}$.

Wright proved the result in the polyhedral category. Metzler and his students have established the CW case (unpublished; see [7, p. 293, Note (2)]). Both Wright and Metzler used transient deformation in their proofs of sufficiency, i.e., a given formal deformation must be modified so that every 3-cell is collapsed immediately after it is introduced.

\section{GeNerALIZED GROUP PRESENTATIONS}

2.1 Word systems. Let $G$ be a group. A $G$-crossed module is a group $\Lambda$ together with an action of $G$ on $\Lambda$ and a homomorphism $d: \Lambda \rightarrow G$ such that

(a) $\lambda_{1} \lambda_{2} \lambda_{1}^{-1}=d\left(\lambda_{1}\right) \cdot \lambda_{2}$ for $\lambda_{i} \in \Lambda$, and

(b) $d(g \cdot \lambda)=g d(\lambda) g^{-1}$ for $g \in G, \lambda \in \Lambda$.

Crossed modules were invented by J. H. C. Whitehead [14] to express the structure of second relative homotopy groups of complexes. See [10] for a general exposition of the properties of crossed modules. We observe that an $R$-module $M$ is always an $R$-crossed module with trivial homomorphism $d$. However, the groups $G$ and $\Lambda$ for a crossed module need not be abelian.

Maps $f=\left(f_{\Lambda}, f_{G}\right):(\Lambda, G, \cdot, d) \rightarrow\left(\Lambda^{\prime}, G^{\prime}, .^{\prime}, d^{\prime}\right)$ between crossed modules are defined in the obvious way. A free crossed module is a free object in the category of crossed modules. Whitehead [14] gave the following construction of a free crossed module. Let $\mathbf{F}(X)$ denote the free group on the set $X$. Choose elements $g_{\alpha}(\alpha \in \mathscr{I})$ of $G$, and consider the subgroup $P$ of $\mathbf{F}(G \times \mathscr{I})$ defined by

$$
P=\text { n.cl. }\left\{(g, \alpha)(h, \beta)(g, \alpha)^{-1}\left(g g_{\alpha} g^{-1} h, \beta\right)^{-1} \mid g, h \in G ; \alpha, \beta \in \mathscr{I}\right\} .
$$

Then the quotient $\Lambda(\mathscr{I})=\mathbf{F}(G \times \mathscr{I}) / P$, together with the $G$-action induced by $g \cdot\left(g^{\prime}, \alpha\right)=\left(g g^{\prime}, \alpha\right)$ and the homomorphism $d: \Lambda(\mathscr{I}) \rightarrow G$ induced by $(g, \alpha) \rightarrow g g_{\alpha} g^{-1}$, forms a free $G$-crossed module. We call $\Lambda(\mathscr{I})$ the standard free $G$-crossed module with basis $\{(1, \alpha) \mid \alpha \in \mathscr{I}\}$ and boundaries $g_{\alpha}$ 
$(\alpha \in \mathscr{I})$. Elements of the subgroup $P$ are called Peiffer identities. Elements $\langle w\rangle \in \Lambda(\mathscr{I})$ are cosets of words of the form

$$
w=\left(g_{1}, \alpha_{1}\right)^{\varepsilon_{1}}\left(g_{2}, \alpha_{2}\right)^{\varepsilon_{2}} \cdots\left(g_{n}, \alpha_{n}\right)^{\varepsilon_{n}}
$$

where $\varepsilon_{i}= \pm 1$.

Let $\mathbf{Z} G$ be the integral group ring of $G$. Suppose $M(\mathscr{I})$ is a free $G$-module with basis $\left\{m_{\alpha} \mid \alpha \in \mathscr{I}\right\}$, and let $(g, \alpha)$ denote the element $g \cdot m_{\alpha} \in M(\mathscr{I})$. Then we may represent the elements $\langle w\rangle \in M(\mathscr{I})$ by words of the form

$$
w=\varepsilon_{1}\left(g_{1}, \alpha_{1}\right)+\varepsilon_{2}\left(g_{2}, \alpha_{2}\right)+\cdots+\varepsilon_{m}\left(g_{m}, \alpha_{m}\right)
$$

where $\varepsilon_{i}= \pm 1, g_{i} \in G, \alpha_{i} \in \mathscr{I}$.

A syllable is an element $\left(g_{1}, \alpha_{1}\right)^{\varepsilon_{1}}$ or $\varepsilon_{i}\left(g_{i}, \alpha_{i}\right)$ appearing in words of the form (2.1.1) or (2.1.2). For convenience, we sometimes use the additive form (2.1.2) rather than the multiplicative form (2.1.1) for words that represent elements of a crossed module, even though crossed modules are not commutative in general.

For an $m$-cell $e^{m}$ in a pointed CW complex pair $(X, A, *)$, let $\phi^{m}$ be a characteristic map for $e^{m}$ and let $\xi^{m}$ be a path from $*$ to the basepoint of $e^{m}$. Theorems (2.1.3) and (2.1.4) below relate free crossed modules and free modules to relative homotopy groups.

Theorem (2.1.3). (See [14, §16] and [2].) Suppose that $X$ is obtained from $a$ connected topological space $A$ by adjoining 2-cells $e_{1}^{2}, \ldots, e_{J}^{2}$.

Then the relative homotopy group $\pi_{2}(X, A)$ is a free $\pi_{1}(A)$-crossed module, and there is an isomorphism of free $\pi_{1}(A)$-crossed modules

$$
\sigma_{2}: \pi_{2}(X, A) \cong \Lambda(\{1, \ldots, J\})
$$

defined by $\sigma_{2}\left(\left[\xi_{j}^{2} \# \phi_{j}^{2} \# \overline{\xi_{j}^{2}}\right]\right)=\langle(1, j)\rangle$.

Theorem (2.1.4). (See [14, $\S 16]$ and [13, V.1].) Suppose that $X$ is obtained from a connected topological space $A$ by adjoining $m$-cells $e_{1}^{m}, \ldots, e_{J}^{m}$ for some dimension $m \geq 3$.

Then the relative homotopy group $\pi_{m}(X, A)$ is a free $\mathbf{Z} \pi_{1}(A)$-module, and there is an isomorphism of free $\mathrm{Z} \pi_{1}(A)$-modules

$$
\sigma_{m}: \pi_{m}(X, A) \cong M(\{1, \ldots, J\})
$$

defined by $\sigma_{m}\left(\left[\xi_{j}^{m}\right] \cdot\left[\phi_{j}^{m}\right]\right)=\langle(1, j)\rangle$.

The isomorphisms of these theorems will be called Whitehead isomorphisms.

We wish to generalize the notion of a finite group presentation by permitting more than two fields. The entries in the new fields will be words $w_{\sigma}^{s}$ $(s>2)$ representing elements in a free module or free crossed module. Such words may correspond to boundaries of higher dimensional cells $e_{\sigma}^{s}$ of a $\mathrm{CW}$ complex, much as relators correspond to the boundaries of 2-cells in a cellular presentation.

Definition (2.1.5). A Peiffer-Whitehead word system or extended group presentation $\mathscr{W}$ consists of a finite list of finite sets $\left\langle\mathscr{W}^{(1)}\left|\mathscr{W}^{(2)}\right| \cdots \mid \mathscr{W}^{(n)}\right\rangle$ together with boundary homomorphisms $d_{3}, \ldots, d_{n}$, described as follows.

$$
\mathscr{W}^{(1)}=\mathscr{I}_{1} \text { is a set of indices, called } 1 \text {-indices. }
$$


$\mathscr{W}^{(2)}=\left\{w_{\beta}^{2} \mid \beta \in \mathscr{I}_{2}\right\}$ is a set of words representing elements of the free group $F=\mathbf{F}\left(\mathscr{I}_{1}\right)$.

$\mathscr{W}^{(3)}=\left\{w_{\gamma}^{3} \mid \gamma \in \mathscr{J}_{3}\right\}$ is a set of words representing elements of the standard free $F$-crossed module $\Lambda=\Lambda\left(\mathscr{I}_{2}\right)$ with boundaries $\left\{c_{\beta}=\left\langle w_{\beta}^{2}\right\rangle \mid \beta \in \mathscr{J}_{2}\right\}$.

$\mathscr{W}^{(m)}=\left\{w_{\mu}^{m} \mid \mu \in \mathscr{I}_{m}\right\} \quad(4 \leq m \leq n)$ is a set of words representing elements of the standard free $\mathrm{Z} G$-module $M_{m}=M_{m}\left(\mathscr{J}_{m-1}\right)$, where $G=F /$ n.cl. $\left(\left\{\left\langle w_{\beta}^{2}\right\rangle \mid \beta \in \mathscr{I}_{2}\right\}\right)$ is the group presented by $\left\langle\mathscr{W}^{(1)} \mid \mathscr{W}^{(2)}\right\rangle$.

$d_{3}: \Lambda\left(\mathscr{I}_{2}\right) \rightarrow F$ is a group homomorphism determined by $d_{3}\langle(1, \beta)\rangle=$ $\left\langle w_{\beta}^{2}\right\rangle$.

$d_{4}: M_{4}\left(\mathscr{J}_{3}\right) \rightarrow \Lambda$ is a homomorphism determined by $d_{4}\langle(1, \gamma)\rangle=$ $\left\langle w_{\gamma}^{3}\right\rangle$, as described below.

$d_{m}: M_{m}\left(\mathscr{J}_{m-1}\right) \rightarrow M_{m-1} \quad(4<m \leq n)$ is a module homomorphism determined by $d_{m}\langle(1, \lambda)\rangle=\left\langle w_{\lambda}^{m-1}\right\rangle$.

We require that all words have trivial boundaries

$$
d_{m}\left\langle w_{\mu}^{m}\right\rangle=\text { identity }\left(\mu \in \mathscr{J}_{m}, 3 \leq m \leq n\right) .
$$

If $\mathscr{W}$ is such a word system, the set $\mathscr{W}^{(m)}$ is called the $m$-field of $\mathscr{W}$, and its elements $w_{\mu}^{m}$ are called $m$-words or words of order $m$. The boundary homomorphisms $d_{m}(m>4)$ are well-defined $\mathrm{Z} G$-maps because $M_{m}$ is free. The map $d_{3}$ coincides with the group homomorphism in the definition of a crossed module. The function $d_{4}: M_{4}\left(\mathscr{J}_{3}\right) \rightarrow \Lambda\left(\mathscr{F}_{2}\right)$ is a homomorphism of the underlying groups $d_{4}:\left|M_{4}\right| \rightarrow|\Lambda|$ that satisfies

$$
d_{4}(g \cdot y)=x \cdot d_{4}(y)
$$

where $y \in M_{4}, g \in G$, and $x \in F$ is any coset representative for $g \in G$. Such a function $d_{4}$ is clearly well-defined. When we describe $d_{4}$ by the equation $d_{4}\langle(1, \gamma)\rangle=\left\langle w_{\gamma}^{3}\right\rangle$ in Definition (2.1.5), we mean $d_{4}\langle(g, \gamma)\rangle=x \cdot\left\langle w_{\gamma}^{3}\right\rangle$ where $x \in F$ represents the coset $g \in G$.

We often write $\mathscr{W}=\left\langle\mathscr{W}^{(1)}\left|\mathscr{W}^{(2)}\right| \cdots \mid \mathscr{W}^{(n)}\right\rangle$ in discussions that do not refer to the boundary homomorphisms directly. Such a word system $\mathscr{W}$ is said to have order $n$. We sometimes write $M_{m}(\mathscr{W})$ to refer to the module $M_{m}\left(\mathscr{J}_{m-1}\right)$ $(m>3)$ or $\Lambda\left(\mathscr{I}_{2}\right) \quad(m=3)$ associated with a word system.

Two word systems

$$
\mathscr{W}=\left\langle\left\{w_{\alpha}^{1}\right\}_{\alpha \in \mathcal{F}_{1}}|\cdots|\left\{w_{\nu}^{n}\right\}_{\nu \in \mathcal{F}_{n}}\right\rangle \text { and } \overline{\mathscr{W}}=\left\langle\left\{\bar{w}_{\bar{\alpha}}^{1}\right\}_{\bar{\alpha} \in \overline{\mathcal{I}}_{1}}|\cdots|\left\{\bar{w}_{\bar{\nu}}^{n}\right\}_{\bar{\nu} \in \overline{\mathscr{I}}_{n}}\right\rangle
$$

have corresponding index sets if there are bijections $\mathscr{I}_{m} \leftrightarrow \bar{I}_{m}$ of the index sets for each order $1 \leq m \leq n$. Such word systems are congruent, $\mathscr{W} \equiv \overline{\mathscr{W}}$, if they have corresponding index sets and each word $\bar{w}_{\bar{\mu}}^{m}$ in $\overline{\mathscr{W}}$ arises from the corresponding word $w_{\mu}^{m}$ of $\mathscr{W}$ by substituting according to those bijections. We also say that $\overline{\mathscr{W}}$ is obtained from $\mathscr{W}$ by reindexing. Congruent word systems are considered to be identical.

2.2 Homotopy reading. The following procedure shows how to associate a word system $\mathscr{W}$ with the structure of any finite connected $\mathrm{CW}$ complex $K$ of dimension $n$. Such a word system $\mathscr{W}$ is called a homotopy reading of $K$. 
When $n=2$, a homotopy reading is a cellular presentation. We also say that $K$ is a geometric realization of $\mathscr{W}$.

1. Identify a maximal tree in $K^{1}$ to a point.

2. Select basepoints $*_{\mu}^{m} \in \operatorname{bdy}\left(e_{\mu}^{m}\right)$ for each cell $e_{\mu}^{m}\left(\mu \in \mathscr{I}_{m}, 2 \leq m \leq n\right)$.

3. Choose connecting paths $\xi_{\mu}^{m}: e^{0} \rightsquigarrow *_{\mu}^{m}$ in $K^{m-1}$.

4. Choose a characteristic map $\phi_{\mu}^{m}: B^{m} \rightarrow \operatorname{cl}\left(e_{\mu}^{m}\right)$ for each cell. These determine orientations on the cells.

We may now define Whitehead isomorphisms $\sigma_{i}$ as follows:

$\sigma_{1}: \pi\left(K^{1}\right) \cong F\left(\mathscr{I}_{1}\right), \sigma_{1}\left(\left[\phi_{\alpha}^{1}\right]\right)=\langle\alpha\rangle$, an isomorphism of free groups,

$\sigma_{2}: \pi_{2}\left(K^{2}, K^{1}\right) \cong \Lambda\left(\mathscr{I}_{2}\right), \sigma_{2}\left(\left[\xi_{\beta}^{2}\right] \cdot\left[\phi_{\beta}^{2}\right]\right)=\langle(1, \beta)\rangle$, so that $\left(\sigma_{1}, \sigma_{2}\right)$ is an isomorphism of free crossed modules, and

$\sigma_{m}: \pi_{m}\left(K^{m}, K^{m-1}\right) \cong M_{m+1}\left(\mathscr{J}_{m}\right), \sigma_{m}\left(\left[\xi_{\mu}^{m}\right] \cdot\left[\phi_{\mu}^{m}\right]\right)=\langle(1, \mu)\rangle$, an isomorphism of free modules $(3 \leq m \leq n)$.

5. Let $w_{\mu}^{m}$ be any word representing the element

$$
\left\langle w_{\mu}^{m}\right\rangle= \begin{cases}\sigma_{1}\left(\left[\xi_{\mu}^{2} \# \partial \phi_{\mu}^{2} \# \overline{\xi_{\mu}^{2}}\right]\right) \in F\left(\mathscr{I}_{1}\right) & \left(\mu \in \mathscr{I}_{2}, m=2\right), \\ \sigma_{m-1}\left(\left[\xi_{\mu}^{m}\right] \cdot\left[\partial \phi_{\mu}^{m}\right]\right) \in M_{m}\left(I_{m-1}\right) \text { or } \Lambda\left(\mathscr{I}_{2}\right) & \left(\mu \in \mathscr{I}_{m}, 3 \leq m \leq n\right) .\end{cases}
$$

6. Finally, define boundary maps $d_{m+1}: M_{m+1}\left(\mathscr{I}_{m}\right) \rightarrow M_{m}\left(\mathscr{I}_{m-1}\right)$ by $d_{m+1}\langle(1, \mu)\rangle=\left\langle w_{\mu}^{m}\right\rangle$, where $M_{3}$ and $M_{2}$ are understood to mean $\Lambda$ and $F$, respectively.

The following relations are easily checked $\left(\partial_{m}\right.$ is the usual boundary operator in relative homotopy).

$$
\begin{aligned}
d_{3} \circ \sigma_{2} & =\sigma_{1} \circ \partial_{2}: \pi_{2}\left(K^{2}, K^{1}\right) \rightarrow F\left(\mathscr{I}_{1}\right), \\
d_{4} \circ \sigma_{3} & =\sigma_{2} \circ \partial_{3}: \pi_{3}\left(K^{3}, K^{2}\right) \rightarrow \Lambda\left(\mathscr{I}_{2}\right), \\
d_{m+1} \circ \sigma_{m} & =\sigma_{m-1} \circ \partial_{m}: \pi_{m}\left(K^{m}, K^{m-1}\right) \rightarrow M_{m}\left(\mathscr{I}_{m-1}\right) \quad(4 \leq m \leq n-1) .
\end{aligned}
$$

Thus

$$
d_{m}\left\langle w_{\mu}^{m}\right\rangle=d_{m} d_{m+1} \sigma_{m}\left(\left[\xi_{\mu}^{m}\right] \cdot\left[\phi_{\mu}^{m}\right]\right)=\sigma_{m-2} \partial_{m-1} \partial_{m}\left(\left[\xi_{\mu}^{m}\right] \cdot\left[\phi_{\mu}^{m}\right]\right)=0 \in M_{m-1} .
$$

This verifies that $\mathscr{W}=\left(\left\langle\left\{w_{\alpha}^{1}\right\}\left|\left\{w_{\beta}^{2}\right\}\right| \cdots \mid\left\{w_{\nu}^{n}\right\}\right\rangle,\left\{d_{m}\right\}\right)$ is a word system.

The correspondence between a homotopy reading and a geometric realization is completely determined by the choices 1 to 5 above. We refer to these selections as homotopy reading choices. If a word $w$ and an attaching map $f=\partial \phi$ are related according to $(2.2 .1)$ above, we say that $w$ is associated with $f, w \sim f$.

As was noted in Subsection 1.3, every group presentation $P$ is a cellular presentation of some 2-complex $K(\mathscr{P})$. Thus, every word system $\mathscr{W}=\left\langle\mathscr{W}^{(1)}\right|$ $\left.\mathscr{W}^{(2)}\right\rangle$ of order 2 has a geometric realization. However, Whitehead's study of homotopy systems shows that higher-order words systems may fail to have geometric realizations.

Example (2.2.3) [14, §15]. The word system

$$
\begin{gathered}
\mathscr{W}=\left\langle x\left|w_{a}^{2}, w_{b}^{2}\right| w_{c}^{3}\left|w_{d}^{4}\right| w_{e}^{5}\right\rangle, \\
w_{a}^{2}=x x, \quad w_{b}^{2}=1, \quad w_{c}^{3}=(\langle x\rangle, a)(1, a)^{-1}, \\
w_{d}^{4}=(\bar{x}, c)+(1, c), \quad w_{e}^{5}=(\bar{x}, d)-(1, d)
\end{gathered}
$$


has no geometric realization, where $\bar{x}$ is the element in $G=F / R$ represented by $\langle x\rangle \in F$.

Such "phantom complexes" arise when a boundary condition $d_{n}\left(\left\langle w^{n}\right\rangle\right)=0$ corresponds to a triviality in relative homotopy that is not a triviality in absolute homotopy (cf. (2.2.2) and Proposition (3.2.3)). Whitehead's work implies the following results about geometric realization.

Proposition (2.2.4) [14, Theorem 2, p. 465]. Every word system of order $n \leq 4$ has a geometric realization.

Proposition (2.2.5) [14, Theorem 3, p. 466]. If $\mathscr{W}$ is a word system for which the group $G(\mathscr{W})$ presented by $\left\langle\mathscr{W}^{(1)} \mid \mathscr{W}^{(2)}\right\rangle$ is trivial, then $\mathscr{W}$ has a geometric realization.

2.3 Equivalence relations. Let $\mathscr{W}$ and $\overline{\mathscr{W}}$ be word systems. Denote the words, index sets and boundary maps of $\mathscr{W}$ by $w_{\mu}^{m}, \mathscr{I}_{m}$, and $d_{m}$, and denote those constituents of $\overline{\mathscr{W}}$ by $\bar{w}_{\mu}^{m}, \overline{\mathscr{I}}_{m}$, and $\bar{d}_{m}$.

In Definition (2.3.5), we introduce three equivalence relations on word systems in the spirit of the EN-equivalence relation on presentations. Some complications arise because moves such as product equivalence of low-order words may lead to spelling changes in higher-order words. N.B. For convenience, we use the additive notation (2.1.2) here even in the case $m=3$.

Definition (2.3.1). The move [ $\mathscr{W}, \overline{\mathscr{W}}]$ is an elementary 1-product equivalence if the following conditions hold for some 1-indices $\alpha, \tau \in \mathscr{I}_{1}$ and $\bar{\alpha} \in \overline{\mathscr{I}}_{1}$.

(1) $\overline{\mathscr{I}_{1}}=\mathscr{I}_{1} \cup\{\bar{\alpha}\}-\{\alpha\}$, and $\overline{\mathscr{I}_{m}}=\mathscr{I}_{m} \quad(m \geq 2)$.

(2) Each 2-word $\bar{w}_{\beta}^{2} \in \overline{\mathscr{W}}$ is obtained from the corresponding 2-word $w_{\beta}^{2} \in$ $\mathscr{W}$ by replacing each occurrence of $\alpha$ by $\bar{\alpha} \tau^{-1}$.

(3) For each $m$-word $w_{\mu}^{m}=\varepsilon_{1}\left(g_{1}, \lambda_{1}\right)+\cdots+\varepsilon_{Q}\left(g_{Q}, \lambda_{Q}\right) \in \mathscr{W} \quad(m \geq 3)$, the corresponding word $\bar{w}_{\mu}^{m} \in \mathscr{W}$ has the form $\bar{w}_{\mu}^{m}=\varepsilon_{1}\left(\bar{g}_{1}, \lambda_{1}\right)+\cdots+$ $\varepsilon_{Q}\left(\bar{g}_{Q}, \lambda_{Q}\right)$, where a representing word for the element $\bar{g}_{q}$ arises from a representing word for the element $g_{q}$ by replacing each occurrence of $\alpha$ by $\bar{\alpha} \tau^{-1}$.

Definition (2.3.2). For $m>1$, the move [ $\mathscr{W}, \overline{\mathscr{W}}$ ] is an elementary m-product equivalence if the following conditions hold for $m$-indices $\mu, \tau \in \mathscr{I}_{m}$ and $\bar{\mu} \in \overline{\mathscr{I}}_{m}$.

(1) $\overline{\mathscr{I}}_{m}=\mathscr{I}_{m} \cup\{\bar{\mu}\}-\{\mu\}$, and $\overline{\mathscr{I}}_{j}=\mathscr{I}_{j}(j \neq m)$.

(2) $\bar{w}_{\bar{\mu}}^{m}=w_{\mu}^{m}+w_{\tau}^{m}$.

(3) Each $(m+1)$-word $\bar{w}_{\xi}^{m+1} \in \overline{\mathscr{W}}\left(\xi \in \mathscr{I}_{m+1}\right)$ arises from $w_{\xi}^{m+1} \in \mathscr{W}$ by replacing every $\mu$-syllable $\varepsilon_{q}\left(g_{q}, \mu\right)$ by $\varepsilon_{q}\left(g_{q}, \bar{\mu}\right)-\varepsilon_{q}\left(g_{q}, \tau\right)$.

(4) $\bar{w}_{\sigma}^{p}=w_{\sigma}^{p}$ for all $p, \sigma \in \mathscr{J}_{p}$ except for the cases above.

Definition (2.3.3). Suppose $m>1$. The move [ $\mathscr{W}, \overline{\mathscr{W}}]$ is an elementary $m$ expansion, $\mathscr{W} \nearrow_{e, m} \overline{\mathscr{W}}$, if there are an $m$-index $\mu \in \mathscr{I}_{m}$ and an $(m-1)$-index $\lambda \in \mathscr{I}_{m-1}$ such that

(1) $\lambda$ occurs exactly once in $w_{\mu}^{m}$, and

(2) $\mathscr{W}$ is congruent to $\overline{\mathscr{W}}-\left\{w_{\mu}^{m}, w_{\lambda}^{m-1}\right\}$. 
We also say that $[\overline{\mathscr{W}}, \mathscr{W}]$ is an elementary $m$-collapse, $\overline{\mathscr{W}} \underset{e, m}{\searrow} \mathscr{W}$. The index $\lambda$ is called the expansion face index (or collapse face index).

Definition (2.3.4). The elementary $P_{n}$-moves are:

$P_{n} 1$. Word-representative equivalence. Replace one word $w_{\mu}^{m}(m \geq 2)$ by a word $\bar{w}_{\mu}^{m}$ such that $\left\langle w_{\mu}^{m}\right\rangle=\left\langle\bar{w}_{\mu}^{m}\right\rangle \in M_{m}$.

$P_{n} 2$. Conjugation. Let $\alpha \in \mathscr{I}$. If $m=2$, replace $w_{\beta}^{2}$ by $\alpha w_{\beta}^{2} \alpha^{-1}$. If $m>$ 2 , replace one word $w_{\mu}^{m}$ by $\langle\alpha\rangle \cdot w_{\mu}^{m}$. Make appropriate adjustments in higher order words.

$P_{n} 3$. Inversion. Replace one word $w_{\mu}^{n}(m \geq 2)$ by the inverted word $\left(w_{\mu}^{m}\right)^{-1}$ $(m=2,3)$ or $-w_{\mu}^{m}(m>3)$. Make appropriate adjustments in higher order words.

$P_{n} 4$. Elementary $m$-expansion or $m$-collapse, where $2 \leq m \leq n$ (2.3.3).

$P_{n}$ 5. Elementary n-product equivalence (2.3.1) or (2.3.2).

Definition (2.3.5). Let $\mathscr{W}$ and $\overline{\mathscr{W}}$ be two word systems.

(a) $\mathscr{W}$ and $\overline{\mathscr{W}}$ are $P_{n}$-equivalent if there is a sequence of elementary $P_{n}$ moves that transforms $\mathscr{W}$ to $\overline{\mathscr{W}}$.

(b) $\mathscr{W}$ and $\overline{\mathscr{W}}$ are fully $P_{n}$-equivalent if $\overline{\mathscr{W}}$ can be obtained from $\mathscr{W}$ by a finite sequence of moves of types $P_{n} 1, \ldots, P_{n} 4$ and elementary $m$-product equivalences of any order $1 \leq m \leq n$.

(c) $\mathscr{W}$ and $\overline{\mathscr{W}}$ are $D_{n}$-equivalent if $\overline{\mathscr{W}}$ can be obtained from $\mathscr{W}$ by a finite sequence of moves of types $P_{n} 3$ and $P_{n} 4$ only.

These equivalence relations are summarized in Table (2.3.6).

TABLE (2.3.6). Equivalence relations among word systems

\begin{tabular}{|c|ccc|}
\hline \multirow{2}{*}{ Move } & \multicolumn{3}{|c|}{ Orders $m$ permitted for } \\
& $P_{n}$ & full $P_{n} \quad D_{n}$ \\
\hline word-representative & $2 \leq m \leq n \quad 2 \leq m \leq n$ & - \\
conjugation & $2 \leq m \leq n \quad 2 \leq m \leq n$ \\
inversion & $2 \leq m \leq n \quad 2 \leq m \leq n \quad 2 \leq m \leq n$ \\
elementary expansion & $2 \leq m \leq n \quad 2 \leq m \leq n \quad 2 \leq m \leq n$ \\
elementary collapse & $2 \leq m \leq n \quad 2 \leq m \leq n \quad 2 \leq m \leq n$ \\
produce equivalence & $m=n \quad 1 \leq m \leq n \quad-$ \\
\hline \multicolumn{2}{|c|}{ Note: Reindexing yields congruent word systems, } \\
which are considered the same
\end{tabular}

A sequence of word systems $\mathscr{W}_{1}, \mathscr{W}_{2}, \ldots, \mathscr{W}_{K}$ will be called a $P_{n}$-sequence if each elementary move $\left[\mathscr{W}_{k}, \mathscr{W}_{k+1}\right]$ is a $P_{n}$-move. We define full $P_{n}$-sequence and $D_{n}$-sequence similarly. If every such move is an elementary expansion or collapse, we write $\mathscr{W}_{1} \wedge \mathscr{W}_{K}$. In that case, the notations $\mathscr{W}_{1} \nearrow \mathscr{W}_{K}, \mathscr{W}_{1} \searrow \mathscr{W}_{K}$, $\mathscr{W}_{k}^{n} \wedge \mathscr{W}_{K}$, etc., are used by analogy with formal deformations of $C W$ complexes. If $\dot{\mathscr{W}}_{1}$ and $\mathscr{W}_{K}$ have order $n$, we say that a $D_{n+1}$-sequence $\mathscr{W}_{1}, \ldots, \mathscr{W}_{K}$ is transient if every $(n+1)$-word is collapsed immediately after it is expanded. 
Lemma (2.3.7). Suppose that $K$ is a $C W$ complex. Let $\mathscr{W}$ be a homotopy reading of $K$ for which a maximal tree $T \subset K^{1}$ is identified, and let $\bar{T}$ be another maximal tree in $K^{1}$.

Then there is a homotopy reading $\overline{\mathscr{W}}$ of $K$ for which $\bar{T}$ is identified such that $\mathscr{W}$ can be transformed into $\overline{\mathscr{W}}$ by a finite sequence of 1-product equivalences and 1-inversions.

Furthermore, each index $\alpha$ occurs the same number of times in each word $w \in \mathscr{W}$ as in the corresponding word $\bar{w} \in \overline{\mathscr{W}}$, unless $\alpha$ is a 1-index represented in one tree and not in the other.

Proof. Generalize $[11,4.6]$ to word systems.

Proposition (2.3.8). If $\mathscr{W}$ and $\overline{\mathscr{W}}$ are two homotopy readings for the same complex $K$, then $\mathscr{W}$ can be transformed to $\overline{\mathscr{W}}$ using word-representative equivalence, conjugation, inversion and elementary 1-product equivalence.

Proof. By the lemma, we may assume the same choice of maximal tree for both readings. We consider the remaining homotopy reading choices of Subsection 2.2. Changes of cell basepoints lead to conjugations. Changes of connecting paths also lead to conjugations. Proposition (1.2.1) implies that we may assume the same characteristic maps after conjugations and inversions. Word-representative equivalence allows us to choose the same words for both homotopy readings. Thus, we can transform $\mathscr{W}$ to a word system $\mathscr{W}^{\prime}$ that is a homotopy reading for $K$ using all of the same choices as for $\overline{\mathscr{W}}$. It follows that $\mathscr{W}^{\prime}=\overline{\mathscr{W}}$.

\section{PROPERTIES OF HOMOTOPY READING}

3.1 Multiple collapse maps and geometric words. If $e^{m}$ is an $m$-cell that can be collapsed in several different ways, we want to show there exists a word $w^{m}$ associated with $e^{m}$ that expresses all of the collapse conditions simultaneously. Such a word $w^{n}$ is called a geometric word, and can be "read" from a multiple collapse map. The existence of multiple collapse maps for 3-cells requires special attention (Proposition (3.1.2)), and implies a claim made without proof in $[16$, p. 24]. We give a decomposition space argument for that case.

We begin with a lemma.

Lemma (3.1.1). Let $X$ and $Y$ be topological spaces. Suppose that the function $p: X \rightarrow Y$ is either (a) a quotient map or (b) a characteristic map for a cell in $Y$. Let $h: X \rightarrow Z$ be a continuous function such that $h \circ p^{-1}$ is single valued.

Then $h \circ p^{-1}$ is continuous. Furthermore, if $h$ is closed then $h \circ p^{-1}$ is closed.

Proof. This is an application of transgression [5, Chapter VI, 3.2].

Proposition (3.1.2) (Existence of multiple collapse maps in dimension three). Let $e^{3}$ be a 3-cell and let $e_{1}^{2}, e_{2}^{2}, \ldots, e_{J}^{2}$ be 2-cells in a CW complex $K$. Suppose that collapse maps exist for each of the pairs $\left(e^{3}, e_{1}^{2}\right),\left(e^{3}, e_{2}^{2}\right), \ldots,\left(e^{3}, e_{J}^{2}\right)$.

Then a multiple collapse map exists for $\left(e^{3}, e_{1}^{2}, \ldots, e_{J}^{2}\right)$.

Proof. Inductively assume that $\phi_{j}: E^{3} \rightarrow \operatorname{cl}\left(e^{3}\right)$ is a multiple collapse map for $\left(e^{3}, e_{1}^{2}, \ldots, e_{j}^{2}\right)$. We will argue that a multiple collapse map $\phi_{j+1}$ for $\left(e^{3}, e_{1}^{2}, \ldots, e_{j+1}^{2}\right)$ can be obtained from $\phi_{j}$ by modifying it near the preimage 
$\phi_{j}^{-1}\left(e_{j+1}^{2}\right)$, then forming a decomposition space $E^{3} / G$ of $E^{3}$. The nondegenerate elements of the decomposition $G$ will be point-inverses $\phi_{j}^{-1}(x)$ of certain elements $x \in e_{j+1}^{2}$.

Let $S$ be the preimage $S=\phi_{j}^{-1}\left(e_{j+1}^{2}\right) \subset E^{3}$. The following conditions clearly hold.

Condition (3.1.3). For each point $x \in e_{j+1}^{2}$ the point-inverse $\phi_{j}^{-1}(x)$ is connected and does not separate the boundary sphere $\operatorname{bdy}\left(E^{3}\right)$.

We now seek to modify the characteristic map $\phi_{j}$ near $S$ in order to bypass potential complications at the frontier $\operatorname{fr}(S)$.

Let $\Phi: D^{3} \rightarrow \operatorname{cl}\left(e^{3}\right)$ be a collapse map for $\left(e^{3}, e_{j+1}^{2}\right)$ as guaranteed by the hypothesis, defined on a 3-ball $D^{3}$. By definition, the map $\Phi$ restricts to a characteristic map $\rho: D^{2} \rightarrow \operatorname{cl}\left(e_{j+1}^{2}\right)$. The 2-ball $D^{2} \subset \operatorname{bdy}\left(D^{3}\right)$ may be identified with the unit disk $\{(r, \theta) \mid 0 \leq r \leq 1\}$ in $\mathbf{R}^{2}$. Extend the continuous function $\lambda: D^{2} \rightarrow D^{2}$ defined by

$$
\lambda(r, \theta)= \begin{cases}(2 r, \theta) & \text { if } r \leq \frac{1}{2}, \\ (1, \theta) & \text { if } r \geq \frac{1}{2},\end{cases}
$$

to a continuous function $\Lambda: D^{3} \rightarrow D^{3}$ that is a homeomorphism on $\operatorname{int}\left(D^{3}\right)$ such that $\Lambda \mid\left(\operatorname{bdy}\left(D^{3}\right)-\operatorname{int}\left(D^{2}\right)\right)=$ identity. Then the composition $H=\Phi$ 。 $\Lambda \circ \Phi^{-1}: \operatorname{cl}\left(e^{3}\right) \rightarrow \operatorname{cl}\left(e^{3}\right)$ is a well-defined continuous map satisfying

$$
H \mid\left(\operatorname{bdy}\left(e^{3}\right)-e^{2}\right)=\text { identity. }
$$

It follows that there is a well-defined continuous map $\tilde{\phi}_{j}=H \circ \phi_{j}: E^{3} \rightarrow \operatorname{cl}\left(e^{3}\right)$. Because of the nature of $H$, this map $\tilde{\phi}_{j}$ is seen to be a multiple collapse map for $\left(e^{3}, e_{1}^{2}, \ldots, e_{j}^{2}\right)$.

Let $G$ be the decomposition of $R^{3}$ whose nondegenerate elements are the point inverses $f_{j}^{-1}(x)$ for points $x \in A^{2}$, where $A^{2}$ is the ball

$$
A^{2}=\rho\left(\left\{(r, \theta) \mid r \leq \frac{1}{2}\right\}\right) \subset e_{j+1}^{2} .
$$

(Here we emphasize that $f_{j}$ is the original attaching map $\phi_{j} \mid \operatorname{bdy}\left(E^{3}\right)$.) Let $q: E^{3} \rightarrow E^{3} / G$ be the associated quotient map. Then $G$ is an upper semicontinuous decomposition of the separable metric space $E^{3}$, so $E^{3} / G$ is a metric space by a well-known theorem. Furthermore, $E^{3} / G$ is a Hausdorff space, and $E^{3}$ is compact, so $q$ is a closed map. Recalling Condition (3.1.3), we may conclude that $E^{3} / G$ is a 3-ball by [6, Theorem 8]. Now define the function $\phi_{j+1}: E / G \rightarrow \operatorname{cl}\left(e^{3}\right)$ by $\phi_{j+1}(\bar{x})=\tilde{\phi}_{j}(x)$ where $\bar{x}$ is the decomposition element containing $x$. It is easy to show that $\phi_{j+1}$ is well defined. Lemma (3.1.1) implies that the function $\phi_{j+1}=\tilde{\phi}_{j} \circ q^{-1}$ is continuous and closed. In addition, $\phi_{j+1}: E^{3} / G \rightarrow \operatorname{cl}\left(e^{3}\right)$ is clearly a surjection, and $\phi_{j+1} \mid\left(\operatorname{int}\left(E^{3}\right) / G\right)$ is a homeomorphism $\operatorname{int}\left(E^{3}\right) / G \approx \operatorname{int}\left(e^{3}\right)$ because this restriction is a bijection. Therefore $\phi_{j+1}$ restricts to characteristic maps for $e_{1}^{2}, \ldots, e_{j}^{2}$ since the decomposition $G$ is trivial away from $S$. It suffices to show that $\phi_{j+1}$ restricts to a characteristic map for $e_{j+1}^{2}$.

The subspace $b=f_{j}^{-1}\left(A^{2}\right) / G \subset E^{3} / G$ is a closed 2-ball, since $G$ consists exactly of the nondegenerate point-inverses of the continuous closed surjection 
$f_{j} \mid f_{j}^{-1}\left(A^{2}\right): f_{j}^{-1}\left(A^{2}\right) \rightarrow A^{2}$ onto the 2-ball $A^{2}$. Furthermore, the continuous closed surjection $\phi_{j+1}$ is bijective on $\operatorname{int}(b)$ by construction, so $\phi_{j+1} \mid \operatorname{int}(b)$ is a homeomorphism $\operatorname{int}(b) \approx e_{j+1}^{2}$. Thus $\phi_{j+1} \mid b$ is a characteristic map for $e_{j+1}^{2}$.

We next check that if $w$ is an $m$-word representing a map in a $\mathrm{CW} m$ complex $X$, then $w$ also represents the induced map after more $m$-cells are added to $X$.

Proposition (3.1.4). Let $m \geq 2$ and suppose that $K$ is an $m$-complex with $m$ cells $\left\{e_{\mu}^{m} \mid \mu \in \mathscr{I}_{m}\right\}$. Let $\mathscr{A}$ and $\mathscr{B}$ be disjoint subsets of $\mathscr{I}_{m}$ satisfying $\mathscr{A} \cup \mathscr{B}=\mathscr{I}_{m}$, and define subcomplexes $X=K^{m-1} \cup\left\{e_{\alpha}^{m} \mid \alpha \in \mathscr{A}\right\}$ and $Y=K^{m-1} \cup\left\{e_{\beta}^{m} \mid \beta \in \mathscr{B}\right\}$.

Then $\pi_{m}\left(K^{m}, K^{m-1}\right)$ is the free product of $\pi_{m}\left(X, K^{m-1}\right)$ and $\pi_{m}\left(Y, K^{m-1}\right)$ in the category of $\pi_{1}\left(K^{m-1}\right)$-crossed modules.

Proof. This follows from the generalized Seifert-Van Kampen Theorem of [3, Theorem C].

Corollary (3.1.5). With the hypotheses of Proposition (3.1.4), suppose that $\bar{w}$ is a word associated with a map $f: B^{m} \rightarrow X$ via an isomorphism

$$
\sigma_{m, X}: \pi_{m}\left(X, K^{m-1}\right) \rightarrow M_{m+1}(\mathscr{A})
$$

(as in Subsection 2.2). Suppose that the isomorphism $\sigma_{m}: \pi_{m}\left(K^{m}, K^{m-1}\right) \rightarrow$ $M_{m+1}\left(\mathscr{J}_{m}\right)$ satisfies $\sigma_{m} \circ i_{*}=\sigma_{m, X}$, where $i_{*}: \pi_{m}\left(X, K^{m-1}\right) \rightarrow \pi_{m}\left(K^{m}, K^{m-1}\right)$ is induced by inclusion.

Then that same word $\bar{w}$ is also associated with the map incl $\circ: B^{m} \rightarrow K$, that is, $\sigma_{m}([$ incl $\circ f])=\langle\bar{w}\rangle \in M_{m+1}\left(\mathscr{J}_{m}\right)$.

Proposition (3.1.6) (Geometric reading of multiple collapse maps). Let $m \geq 3$, and let $e_{\mu}^{m}$ be a cell in a CW complex $K$. Suppose that a multiple collapse map $\phi_{\mu}^{m}: B \rightarrow \operatorname{cl}\left(e_{\mu}^{m}\right)$ exists for $\left(e_{\mu}^{m}, e_{\alpha_{1}}^{m-1}, \ldots, e_{\alpha_{0}}^{m-1}\right)$.

Then there is a word $w \sim \partial \phi_{\mu}^{m}$ that mentions each $(m-1)$-index $\alpha_{q}$ exactly once.

Proof. Define subcomplexes $X=K^{m-2} \cup\left\{e_{\alpha}^{m-1} \mid \alpha \in \mathscr{A}\right\}$ and $Y=K^{m-2} \cup$ $\left\{e_{\beta}^{m-1} \mid \beta \in \mathscr{B}\right\}$, where $\mathscr{A}=\left\{\alpha_{1}, \ldots, \alpha_{Q}\right\}$ and $\mathscr{B}=\mathscr{I}_{m}-\mathscr{A}$.

Let $\left(B, b_{1}, \ldots, b_{Q}\right)$ be a topological $m$-ball tuple for $\phi_{\mu}^{m}$ such that $\phi_{\mu}^{m} \mid b_{q}$ is a characteristic map for $e_{\alpha_{q}}^{m-1} \quad(1 \leq q \leq Q)$, as guaranteed in the definition of a multiple collapse map. Connect the $(m-1)$-balls $b_{q}$ to the basepoint of $B$ by nonintersecting arcs $a_{q}: I \rightarrow\left(\phi_{\mu}^{m}\right)^{-1}\left(K^{m-2}\right)$. Fatten these arcs slightly to form a closed neighborhood $N$ about $\bigcup_{q}\left\{a_{q} \cup b_{q}\right\}$ so that both $N$ and $\operatorname{cl}(B-N)$ are topological $(m-1)$-balls. We may modify the attaching map $f_{\mu}^{m}$ by a homotopy $f_{\mu}^{m} \simeq f_{1} \quad\left(\mathrm{rel} *_{B}\right)$ so that the image $f_{1}\left(N-\bigcup_{q} b_{q}\right)$ is contained in $K^{m-2}$. By Proposition (1.2.1), the map $f_{1}$ is homotopic to a map $f_{2}$ such that all of the following conditions hold.

(a) For some interior ball $b_{q}^{\prime} \subset \operatorname{int}\left(b_{q}\right)$, the restriction $f_{2} \mid b_{q}^{\prime}$ is a copy of the characteristic map $\phi_{\alpha_{q}}^{2}$, up to reversal.

(b) $f_{2}\left(N-\bigcup_{q}\left(b_{q}^{\prime}\right)\right) \subset K^{m-2}$.

(c) $f_{2}\left|\operatorname{cl}(\dot{B}-N)=f_{1}\right| \operatorname{cl}(\dot{B}-N)$, where $\dot{B}=$ bdy $B$. 
By the definition of the Whitehead isomorphism $\sigma_{m-1}: \pi_{m-1}\left(X, K^{m-2}\right) \rightarrow$ $M_{m}(\mathscr{A})$ of Theorem (2.1.3) or Theorem (2.1.4), the map $f_{2} \mid N: N \rightarrow X$ is associated with a word $w_{1}=\varepsilon_{1}\left(g_{1}, \alpha_{1}\right)+\cdots+\varepsilon_{q}\left(g_{q}, \alpha_{q}\right)$, i.e.,

$$
\left\langle w_{1}\right\rangle=\sigma_{m-1, X}\left(\left[f_{2} \mid N\right]\right) \in M_{m}(\mathscr{A}) .
$$

(When $m=3, w_{1}=\left(\left(g_{1}, \alpha_{1}\right)^{\varepsilon_{1}} \cdots\left(g_{q}, \alpha_{q}\right)^{\varepsilon_{q}}\right.$.) Also, $f_{2}(\mathrm{cl}(\dot{B}-N))$ is contained in the subcomplex $Y$ and has an associated word $w_{2}$ representing an element in $M_{m}(\mathscr{B})$. Such a word $w_{2}$ cannot possibly mention any indices $\alpha_{1}, \ldots, \alpha_{Q}$.

Applying Corollary (3.1.5) to the words $w_{1}$ and $w_{2}$, we see that

$$
\sigma_{m-1}\left(\left[f_{\mu}^{m}\right]\right)=\sigma_{m-1}\left(\left[f_{2}\right]\right)= \begin{cases}\left\langle w_{1}\right\rangle+\left\langle w_{2}\right\rangle=\left\langle w_{1}+w_{2}\right\rangle & \text { when } m>3, \\ \left\langle w_{1}\right\rangle\left\langle w_{2}\right\rangle=\left\langle w_{1} w_{2}\right\rangle & \text { when } m=3 .\end{cases}
$$

Let $w=w_{1}+w_{2}$ if $m>3$, or $w=w_{1} w_{2}$ if $m=3$. Then $w \sim \partial \phi_{\mu}^{m}=f_{\mu}^{m}$, where $w$ is a word that mentions each $(m-1)$-index $\alpha_{1}, \ldots, \alpha_{Q}$ exactly once.

Suppose that $e^{m}$ is an $m$-cell $(m \geq 2)$ in a CW complex $K$, and that $\left(e^{m}, e_{\alpha_{1}}^{m-1}\right), \ldots,\left(e^{m}, e_{\alpha_{Q}}^{m-1}\right)$ is a complete set of pairs for which collapse maps exist. Let $\sigma_{m-1}$ be a Whitehead isomorphism as in Theorem (2.1.3) or Theorem (2.1.4). A word $w^{m}$ is a geometric word for $e^{m}$ if

(a) $w^{m} \sim f^{m}$,

(b) $w^{m}$ mentions the $(m-1)$-indices $\alpha_{1}, \ldots, \alpha_{Q}$ exactly once each, and

(c) all $(m-1)$-indices $\alpha$ mentioned in $w^{m}$ are indices of $(m-1)$-cells $e_{\alpha}^{m-1}$ contained in the carrier subcomplex for $e^{m}$ (i.e., the smallest subcomplex of $K$ containing $\left.\operatorname{cl}\left(e^{m}\right)\right)$.

N.B. When the dimension is $m=2$, condition (b) applies only to 1-indices $\alpha_{q}$ for which $e_{\alpha_{q}}^{1}$ is not part of the maximal tree $T \subset K^{1}$ chosen for the homotopy reading, since 1-cells in $T$ were not assigned indices in $\mathscr{I}_{1}$.

Proposition (3.1.7) (Existence of geometric words). A geomeiric word $w^{m}$ exists for every cell $e^{m}$ of dimension $m \geq 2$ in a CW complex $K$.

Proof. The argument is straightforward when $m=2$. For $m>3$, let $\alpha_{q}$ be among the indices $\alpha_{1}, \ldots, \alpha_{Q}$ referred to in the definition of a geometric word. Proposition (3.1.6) shows that there is a word $w_{q}^{m} \sim f^{m}$ that mentions the index $\alpha_{q}$ exactly once. It follows that any freely reduced word $w^{m} \sim f^{m}$ mentions the index $\alpha_{q}$ exactly once, for all $q(1 \leq q \leq Q)$. We may assume that $w^{m}$ satisfies the carrier condition (c) by Corollary (3.1.5), so $w^{m}$ is a geometric word for $e^{m}$.

When the dimension is $m=3$, freely reduced words are not available. The proposition follows in this case by applying geometric reading (3.1.6) and Corollary (3.1.5) to a multiple collapse map for $e^{m}$, which exists by Proposition (3.1.2).

Corollary (3.1.8). Suppose that $K \nearrow_{e, m} \bar{K}$ where $K$ and $\bar{K}$ are $C W$ complexes, and that $\mathscr{W}$ is a homotopy reading for $K$ consisting entirely of geometric words. Then $\mathscr{W} \nearrow_{e, m} \overline{\mathscr{W}}$, where $\overline{\mathscr{W}}$ is a homotopy reading for $\bar{K}$ consisting entirely of geometric words. 
3.2 Model cells and model complexes. Having found words that reflect the collapse structure of cells, we now seek complexes that geometrically reflect the word structure of a word system. Such specialized complexes will be used in Proposition (3.3.5) to show that any two complexes sharing the same homotopy reading must homotopy $n$-deform to each other.

Suppose that $K$ is a CW complex with one 0 -cell $e^{0}$. A 2-cell $e^{2} \in K$ is a model cell for $w^{2}=\alpha_{1}^{\varepsilon_{1}} \cdots \alpha_{Q}^{\varepsilon_{Q}}$ if there is a subdivision of $S^{1}=\operatorname{bdy}\left(E^{2}\right)$ into consecutive arcs $A_{1}, \ldots, A_{Q}$ such that $\phi^{2} \mid A_{q}$ is a copy of the characteristic map $\phi_{\alpha_{q}}^{1}$ for $e_{\alpha_{q}}^{1}$, with orientation determined by $\varepsilon_{q}$.

We say that a path $\gamma: \mathbf{I} \rightarrow K^{1}$ models an element $x \in F\left(\mathscr{J}_{1}\right)$ if the condition $\phi_{1}([\gamma])=x$ holds. We also say that $\gamma: \mathbf{I} \rightarrow K^{1}$ models an element $g \in G=$ $F\left(\mathscr{J}_{1}\right) / R$ if $\gamma$ models a coset representative $x$ of $g$.

An $m$-cell $e^{m} \in K \quad(m \geq 3)$ with characteristic map $\phi^{m}: E^{m} \rightarrow \operatorname{cl}\left(e^{m}\right)$ is a model cell for $w^{m}=\varepsilon_{1}\left(x_{1}, \lambda_{1}\right)+\cdots+\varepsilon_{Q}\left(x_{Q}, \lambda_{Q}\right)$ if there exist disjoint closed $(m-1)$-balls $B_{1}, \ldots, B_{Q}$ in bdy $\left(E^{m}\right)$ such that the following conditions hold.

(1) $\phi^{m}\left(\operatorname{bdy}\left(E^{m}\right)-\left(\bigcup B_{q}\right)\right) \subset K^{m-2}$.

(2) $\phi^{m} \mid B_{q}$ is a copy of the characteristic map $\phi_{\lambda_{q}}^{m-1}$ for $e_{\lambda_{q}}^{m-1}$, with orientation determined by $\varepsilon_{q}$.

(3) There are paths $\alpha_{q}:\left(*_{E^{m}}\right) \rightsquigarrow\left(*_{B_{q}}\right)$ in bdy $\left(E^{m}\right)$ such that

(a) the union of the images of the paths $\alpha_{q}$ is a tree that meets each ball $B_{q}$ in a unique extreme point, and

(b) $\phi^{m} \circ \alpha_{q}$ is a path product $\phi^{m} \circ \alpha_{q}=\gamma_{q} \# \xi_{\lambda_{q}}^{m-1}$, where $\gamma_{q}: I \rightarrow$ $K^{1}$ models $x_{q}$ and $\xi_{\lambda_{q}}^{m-1}$ is a fixed choice of connecting path $\xi_{\lambda_{q}}^{m-1}:\left(e^{0}\right) \rightsquigarrow\left(*_{\lambda_{q}}^{m-1}\right)$ for each cell $e_{\lambda_{q}}^{m-1}$.

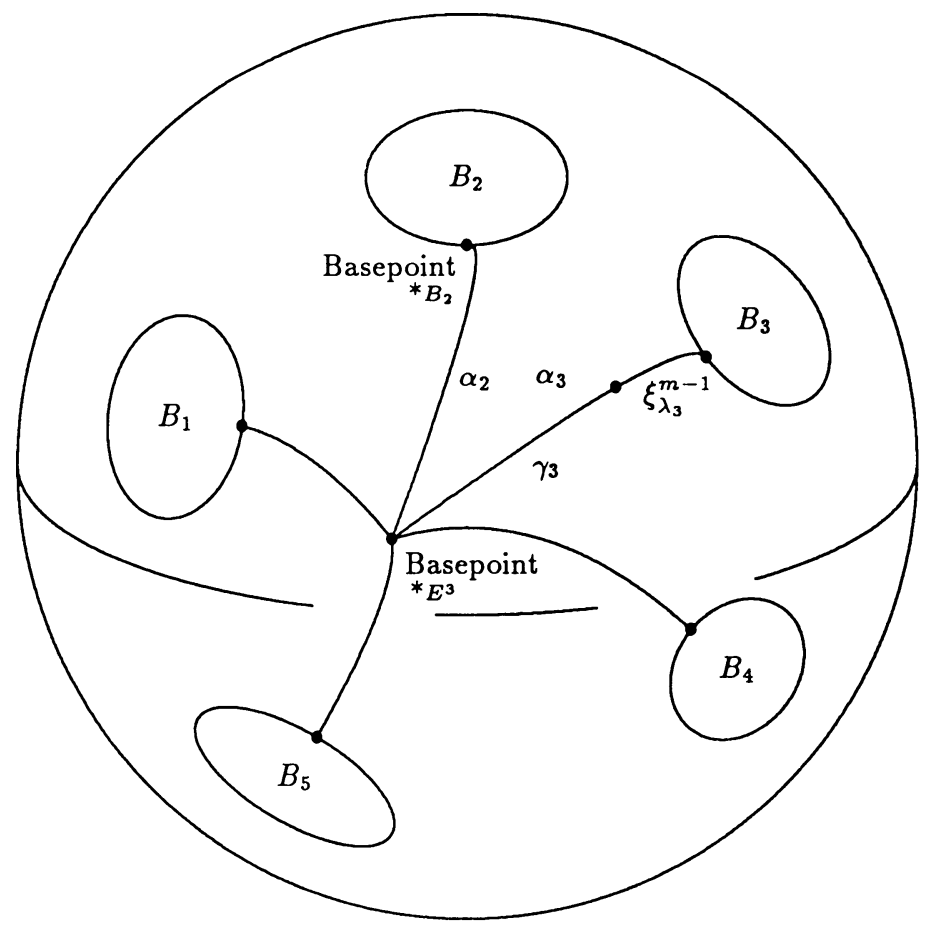

FigURE (3.2.1). Diagram of a model cell for $m=3$ 
When the dimension is $m=3$, we further require that the balls $B_{q}$ be encountered in the natural order $B_{1}, \ldots, B_{Q}$ as one traverses the tree system around the basepoint of $E^{3}$ (see Figure (3.2.1)).

The attaching map $f^{m}=\phi^{m} \mid \mathrm{bdy}\left(E^{m}\right)$ of such a cell is called a model map for $w^{m}$. It is clear that $w^{m} \sim f^{m}$.

A CW complex is a model complex $K(\mathscr{W})$ for a word system $\mathscr{W}$ if $K(\mathscr{W})$ has one 0 -cell and each cell $e_{\mu}^{m} \in K(\mathscr{W})$ is a model cell for the corresponding word $w_{\mu}^{m} \in \mathscr{W}$. Trivially, a model complex $K(\mathscr{W})$ is a geometric realization of $\mathscr{W}$.

Proposition (3.2.2). If $f$ is a model map for a word $w^{m}$ then $f$ is also a model map for any cyclic permutation of $w^{m}$.

Obviously, model maps exist for any 2-word.

Proposition (3.2.3) (Existence of model maps). Let $\mathscr{W}$ be a homotopy reading of a $C W$ complex $K$. Let $w$ be a word, not necessarily in $\mathscr{W}$, that represents an element in $M_{m}(\mathscr{W})(m>2)$. Let

$$
f:\left(I^{m-1}, \operatorname{bdy}\left(I^{m-1}\right), I^{m-2} \times 0\right) \rightarrow\left(K^{m-1}, K^{m-2}, *\right)
$$

be a map such that $w \sim f$ and $\beta([f])=0$, for the homotopy boundary homomorphism $\beta: \pi_{m-1}\left(K^{m-1}, K^{m-2}\right) \rightarrow \pi_{m-2}\left(K^{m-2}\right)$.

Then $f$ is homotopic rel basepoint in $K^{m-1}$ to a model map $f^{*}$ for $w$.

In particular, if $f=f^{m}$ is an attaching map for a cell, then a model map exists for any word $w \sim f^{m}$.

Remarks. (1) Observe that $d_{m}(\langle w\rangle)=1$ follows from the conditions on $f$. (2) We may not assume $a$ priori that a map such as $f$ exists when $m>4$, as demonstrated by the word $w_{e}^{5}$ of Example (2.2.3) for which $\beta([f])=0$ cannot be satisfied.

Proof of Proposition (3.2.3). Write $w$ in the form $w=\varepsilon_{1}\left(g_{1}, \lambda_{1}\right)+\cdots+$ $\varepsilon_{Q}\left(g_{Q}, \lambda_{Q}\right)$. Let $X$ be a subspace of the $(m-1)$-sphere $S^{m-1}$ consisting of $(m-1)$-balls $B_{1}, \ldots, B_{Q}$ and simple, nonintersecting arcs $\alpha_{q}$ joining the basepoint of $S^{m-1}$ to the basepoint of $B_{q}(1 \leq q \leq Q)$. Let $N$ be a neighborhood of $X$ found by thickening $\operatorname{fr}(X)$, such that $N$ and $\operatorname{cl}\left(S^{m-1}-N\right)$ are $(m-1)$-balls.

Let $F: X \rightarrow K^{m-1}$ be a map such that $F \mid B_{q}$ is a copy of the characteristic map $\phi_{\lambda_{q}}^{m-1}$ for some path $\gamma_{q}$ that models the element $g_{q}$. Extend $F$ to a map $\tilde{F}:(N, \operatorname{bdy}(N)) \rightarrow\left(K^{m-1}, K^{m-2}\right)$ by means of a retraction $r$, so that $\widetilde{F}$ is associated with the element $\langle w\rangle$. This means that $\sigma_{m-1}([\widetilde{F}])=\langle w\rangle \in$ $M_{m}(\mathscr{W})$, where $\sigma_{m-1}$ is a Whitehead isomorphism.

A computation using the hypothesis $[f]=\sigma^{-1}(\langle w\rangle)=[\widetilde{F}]$ and $\beta([f])=$ $0 \in \pi_{m-2}\left(K^{m-2}\right)$ shows that $\partial \widetilde{F}$ is nullhomotopic. Use this nullhomotopy to extend $\widetilde{F}$ to a map $F^{*}: S^{m-1} \rightarrow K^{m-1} . F^{*}$ is clearly a model map for $w$, and $\sigma_{m-1}\left(\left[F^{*}\right]\right)=\langle w\rangle=\sigma_{m-1}([f])$ implies that $\left[F^{*}\right]=[f] \in \pi_{m-1}\left(K^{m-1}, K^{m-2}\right)$.

3.3 Geometric dragging and substitution. Let $K$ be an $n$-complex. Geometric dragging is a technique by which an elementary homotopy $m$-deformation of the 
$m$-skeleton of $K(m \leq n)$ is lifted to a homotopy $n$-deformation $K \stackrel{n}{\wedge} L$ of the entire complex $K$. Our terminology is motivated by the following example.

Example (3.3.1). Let $D=e^{0} \cup e^{1} \cup e^{2}$ be a $\mathrm{CW}$ decomposition of a disk, and consider the subcomplex $K_{1}=\left(e^{0} \cup e^{1}\right) \times I$ of $D \times I$. Consider the elementary homotopy 2-move

$$
K=K_{1} \cup\left(e^{2} \times 0\right) \underset{e, 2}{\rightarrow} K_{1} \cup\left(e^{2} \times 1\right)=L
$$

and let $e^{3}$ be the 3-cell of the associated 3-expansion.

Now suppose that $K$ is a subcomplex of a CW complex $X$ that does not include $e^{3}$, and let $e_{0}^{3}$ be a 3-cell in $X$ that has the 2-cell $e^{2} \times 0$ in its boundary. The homotopy move $K \underset{e, 2}{\rightarrow} L$ above could be used to induce a modification of the attaching map of $e_{0}^{3}$, yielding a new 3-cell $e_{1}^{3}$ that has the 2-cells $e^{2} \times 1$ and $e^{1} \times(0,1)$ in its boundary. We describe this as "dragging" $e_{0}^{3}$ to $e_{1}^{3}$.

The effect of that homotopy move on a word $w_{0}^{3}$ for $e_{0}^{3}$ can be described as substitution. For example, suppose that $w^{3}=\left(1, \mu_{0}\right)^{-1}(1, \mu)\left(1, \mu_{1}\right)$ is a geometric word for $e^{3}$ in some homotopy reading of $X \cup(D \times I)$, where $\mu_{i} \in \mathscr{I}_{2}$ is the index for $e^{2} \times i \quad(i=0,1)$ and $\mu \in \mathscr{I}_{2}$ is the index for $e^{1} \times(0,1)$. Then under suitable conditions on $e_{0}^{3}$, every occurrence of the syllable $\left(1, \mu_{0}\right)$ in $w_{0}^{3}$ may be replaced by the word $\hat{w}=(1, \mu)\left(1, \mu_{1}\right)$ to yield a word $w_{1}^{3}$ for $e_{1}^{3}$.

Proposition (3.3.2) (Internal collapse of a maximal tree). Let $T \subset K^{1}$ be $a$ maximal tree in an $n$-complex $K$.

Then $K \stackrel{n}{\Gamma^{\prime}} K / T$.

Proof. Let $F: K \rightarrow K / T$ be the quotient map, and consider the mapping cylinders $M(m)=M\left(F \mid K^{m}\right) \quad(1 \leq m \leq n-1)$ of restrictions of $F$ to the skeletons of $K$. There are expansions

$$
\begin{aligned}
& K{ }^{2} M(1) \cup\left(\bigcup e_{\beta}^{2}\right) \cup\left(\bigcup e_{\gamma}^{3}\right) \cup \cdots \cup\left(\bigcup e_{\nu}^{n}\right) \\
& \quad{ }^{3} M(2) \cup\left(\bigcup e_{\gamma}^{3}\right) \cup \cdots \cup\left(\bigcup e_{\nu}^{n}\right) \\
& \quad{ }^{4} \cdots \\
& \quad{ }^{n} M(n-1) \cup\left(\bigcup e_{\nu}^{n}\right)=K_{1},
\end{aligned}
$$

where $e_{\mu}^{m}(m=2, \ldots, n)$ are cells of $K$. Slide the attaching map of each $n$-cell $e_{\nu}^{n}$ in $K_{1} \quad\left(\nu \in \mathscr{J}_{n}\right)$ along the walls of the mapping cylinder $M(n-1)$ in a level-preserving manner to obtain a homotopy $n$-deformation $K_{1} \stackrel{n}{\rightarrow} K_{2}=$ $M(n-1) \cup\left(\bigcup \bar{e}_{\nu}^{n}\right)$, for $\bar{e}_{\nu}^{n}\left(\nu \in \mathscr{I}_{n}\right)$ as in Definition (1.2.2). Observe that the $n$-cells $\bar{e}_{\nu}^{n} \in K_{2}\left(\nu \in \mathscr{I}_{n}\right)$ are precisely the $n$-cells in $K / T$. Finally, perform cylindrical collapses

$$
\begin{aligned}
& K_{2}=M(n-1) \cup\left(\bigcup \bar{e}_{\nu}^{n}\right) \\
& \searrow_{1}^{n}\left(K^{n-1} / T\right) \cup\left(\bigcup \bar{e}_{\nu}^{n}\right)=K / T .
\end{aligned}
$$


We now turn to the notion of substitution, as motivated by Example (3.3.1). N.B. We use the additive notation (2.1.2) here even in the case $m=3$, for convenience.

Suppose that $m \geq 3$. The boundary equivalence relation $\doteq$ on words of order $m$ is defined by $w_{1} \doteq w_{2}$ if and only if $d_{m}\left(\left\langle w_{1}\right\rangle\right)=d_{m}\left(\left\langle w_{2}\right\rangle\right)$. Suppose that $\mathscr{W}=\left\langle\left\{w_{\alpha}^{1}\right\}|\cdots|\left\{w_{\nu}^{n}\right\}\right\rangle$ is a word system of order $n$, and let $\hat{w}$ be a new $m$-word such that $\hat{w} \doteq\left(1, \lambda_{0}\right)$ for some $\lambda_{0} \in \mathscr{I}_{m-1}$ where $\lambda_{0}$ is not mentioned in $\hat{w}$. We permit $\hat{w}$ to mention new $(m-1)$-indices $\eta_{j} \notin \mathscr{I}_{m-1}(1 \leq j \leq J)$, provided that boundaries $d_{m}\left(1, \eta_{j}\right)=\left\langle\bar{w}_{\eta_{j}}^{m-1}\right\rangle$ are also supplied.

We say that $\overline{\mathscr{W}}=\left\langle\left\{\bar{w}_{\alpha}^{1}\right\}|\cdots|\left\{\bar{w}_{\nu}^{n}\right\}\right\rangle$ arises from $\mathscr{W}$ by substituting $\hat{w}$ for $\lambda_{0}$ if $\overline{\mathscr{W}}$ satisfies the following conditions.

(a) The index sets for $\overline{\mathscr{W}}$ are $\overline{\mathscr{I}}_{m-1}=\mathscr{I}_{m-1}-\left\{\lambda_{0}\right\} \cup\left\{\eta_{1}, \ldots, \eta_{J}\right\}$ and $\overline{\mathscr{I}}_{s}=\mathscr{I}_{s}(s \neq m-1)$.

(b) $\bar{w}_{\sigma}^{s}=w_{\sigma}^{s}$ for $s<m-1, s>m$ or $\left(s=m-1\right.$ and $\left.\sigma \neq \lambda_{0}\right)$.

(c) $\bar{w}_{\mu}^{m}\left(\mu \in \mathscr{I}_{m}\right)$ is formed by replacing each syllable $\varepsilon_{q}\left(g_{q}, \lambda_{0}\right)$ in $w_{\mu}^{m}$ by the word $\varepsilon_{q} g_{q} \cdot \hat{w}$.

Note that the $(m-1)$-word $w_{\lambda_{0}}^{m-1}$ is omitted from $\overline{\mathscr{W}}$.

When $m=2$, the definition of substitution is complicated by the fact that changing the 1 -field can affect the coefficients $g$ in higher-order syllables $\varepsilon(g, \rho)$.

Suppose that $\mathscr{W}=\left\langle\left\{w_{\alpha}^{1}\right\}|\cdots|\left\{w_{\nu}^{n}\right\}\right\rangle$ is a word system of order $n$, and let $\hat{w}$ be a new 2 -word which may mention new 1-indices $\eta_{j} \notin \mathscr{I}_{1}$. Then we say that $\overline{\mathscr{W}}=\left\langle\left\{\bar{w}_{\alpha}^{1}\right\}|\cdots|\left\{\bar{w}_{\nu}^{n}\right\}\right\rangle$ arises from $\mathscr{W}$ by substituting $\hat{w}$ for $\alpha_{0}$ if $\overline{\mathscr{W}}$ satisfies the following conditions.

(a) The index sets for $\overline{\mathscr{W}}$ are $\overline{\mathscr{I}_{1}}=\mathscr{I}_{1}-\left\{\alpha_{0}\right\} \cup\left\{\eta_{1}, \ldots, \eta_{J}\right\}$ and $\overline{\mathscr{I}}_{s}=\mathscr{I}_{s}$ $(s>1)$.

(b) $\bar{w}_{\alpha}^{1}=w_{\alpha}^{1}$ for $\alpha \neq \alpha_{0}$.

(c) $\bar{w}_{\beta}^{2} \quad\left(\beta \in \mathscr{I}_{2}\right)$ is found by replacing each occurrence of $\alpha_{0}^{\varepsilon}$ in $w_{\beta}^{2}$ by the word $(\hat{w})^{\varepsilon} \quad(\varepsilon= \pm 1)$.

(d) $\bar{w}_{\sigma}^{s}\left(s \geq 3, \sigma \in \mathscr{F}_{s}\right)$ is found by replacing each syllable $\varepsilon_{q}\left(g_{q}, \rho_{q}\right)$ in $w_{\sigma}^{s}$ by the syllable $\varepsilon_{q}\left(\tilde{g}_{q}, \rho_{q}\right)$, where a representing word for $\tilde{g}_{q}$ is obtained from a representing word for $g_{q}$ by replacing each occurrence of $\alpha_{0}^{\varepsilon}$ by $(\hat{w})^{\varepsilon} \quad(\varepsilon= \pm 1)$.

Observe that a substitution opportunity occurs whenever there is a word $w^{l}=$ $\varepsilon_{1}\left(g_{1}, \kappa_{1}\right)+\cdots+\varepsilon_{Q}\left(g_{Q}, \kappa_{Q}\right)$ in $\mathscr{W}$ with trivial boundary that mentions an $(l-1)$-index $\kappa$ exactly once. (When $l=3$, the word would have the form $w^{3}=\left(g_{1}, \kappa_{1}\right)^{\varepsilon_{1}} \cdots\left(g_{Q}, \kappa_{Q}\right)^{\varepsilon_{Q}}$. When $l=2, w^{2}=\kappa_{1}^{\varepsilon_{1}} \cdots \kappa_{Q}^{\varepsilon_{Q}}$.) To see this, let

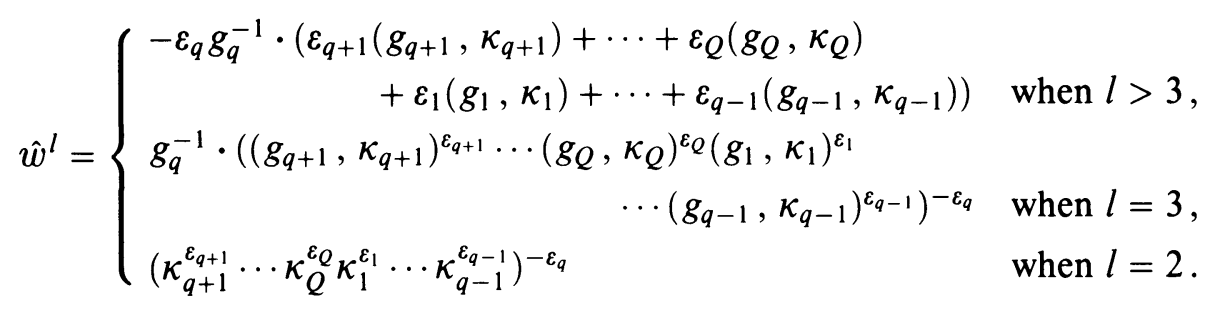

Then it is clear that $\hat{w}^{l} \doteq\left(1, \kappa_{q}\right)$ if $l \geq 3$ and that $\hat{w}^{l}$ does not mention $\kappa_{q}$, so substitution is possible. We say that we are substituting via $w^{l}$. We also say 
that $\hat{w}^{l}$ arises from $w^{l}$ by solving for $\left(1, \kappa_{q}\right)$ (or for $\kappa_{q}$, if $l=2$ ).

Proposition (3.3.3) (Geometric dragging technique). Let $m \geq 1$. Suppose that $K$ is a geometric realization of a word system $\mathscr{W}$ of order $n$ such that each cell $e_{\sigma}^{s} \in K$ of dimension $s>m$ is a model cell for the corresponding word $w_{\sigma}^{s} \in \mathscr{W}$. Let $K^{m} \underset{e, m}{\rightarrow} K^{m} \cup \bar{e}_{\bar{\mu}}^{m}-e_{\mu}^{m}$ be an elementary homotopy m-move of the $m$-skeleton of $K$ with associated expansion cell $\tilde{e}_{\mu}^{m+1}$.

Then $K$ homotopy $n$-deforms to an $n$-complex $\bar{K}$ that satisfies the following conditions.

(a) $\bar{K}^{m}=K^{m} \cup \bar{e}_{\bar{\mu}}^{m}-e_{\mu}^{m}$.

(b) $\bar{K}$ is a geometric realization of the word system $\overline{\mathscr{W}}$ that arises from $\mathscr{W}$ by substitution via $\tilde{w}_{\mu}^{m+1}$, where $\tilde{w}_{\mu}^{m+1}$ is a word for $\tilde{e}_{\mu}^{m+1}$ that mentions the $m$-indices $\mu$ and $\bar{\mu}$ exactly once each.

(c) The cells $\bar{e}_{\sigma}^{s} \in \bar{K} \quad(s>m)$ are model cells for the corresponding words $\bar{w}_{\sigma}^{s} \in \overline{\mathscr{W}}$.

Proof. First, observe that Proposition (3.1.7) guarantees that a geometric word $\tilde{w}_{\mu}^{m+1}$ exists satisfying (b). Thus the word system $\overline{\mathscr{W}}$ is well defined. Note that a model map $\tilde{f}_{\mu}^{m+1}$ for the word $\tilde{w}_{\mu}^{m+1}$ exists, by Proposition (3.2.3).

Let $\dot{\mathbf{I}}^{m+1}$ denote bdy $\left(\mathbf{I}^{m} \times \mathbf{I}\right)$. Adjust $\tilde{f}_{\mu}^{m+1}: \dot{\mathbf{I}}^{m+1} \rightarrow K^{m}$ if necessary so that

(1) $\tilde{f}_{\mu}^{m+1} \mid \mathbf{I}^{m} \times 0$ is a characteristic map for $e_{\mu}^{m}$, and

(2) $\tilde{f}_{\mu}^{m+1} \mid \mathbf{I}^{m} \times 1$ is a characteristic map for $\bar{e}_{\bar{\mu}}^{m}$.

The existence of such characteristic maps is guaranteed by the definition of a model map. Without loss of generality, $\tilde{f}_{\mu}^{m+1}$ may be taken as the attaching $\operatorname{map}$ for $\tilde{e}_{\mu}^{m+1}$.

We will proceed to slide the attaching maps of cells in $K$ across the expansion cell $\tilde{e}_{\mu}^{m+1}$, one dimension at a time, noting the effect on words as we go.

Define the complex

$$
K(m)=K \cup\left\{\bar{e}_{\bar{\mu}}^{m}, \tilde{e}_{\mu}^{m+1}\right\} .
$$

Then the characteristic map $\tilde{\phi}_{\mu}^{m+1}: \mathbf{I}^{m+1} \rightarrow \operatorname{cl}\left(\tilde{e}_{\mu}^{m+1}\right) \subset K(m)$ is the homotopy $\tilde{\phi}_{\mu}^{m+1}: \phi_{\mu}^{m} \simeq \bar{\phi}_{\bar{\mu}}^{m}$ of the homotopy $m$-deformation of $K^{m}$ of the hypothesis. We may define a map $F_{m}: K^{m} \times \mathbf{I} \rightarrow K(m)$ by

$$
F_{m}(x, t)=\left\{\begin{array}{l}
x \text { for } x \in K^{m}-e_{\mu}^{m}, \\
\tilde{\phi}_{\mu}^{m+1} \circ D_{t}\left(\left(\phi_{\mu}^{m}\right)^{-1}(x), 0\right) \text { for } x \in e_{\mu}^{m}
\end{array}\right.
$$

for a strong deformation retraction $D_{t}$ from $\mathbf{I}^{m+1}$ to $\operatorname{cl}\left(\dot{\mathbf{I}}^{m+1}-\left\{\mathbf{I}^{m} \times 0\right\}\right)$. The map $F_{m}$ is continuous by Lemma (3.1.1). As $t$ varies from 0 to 1 , the effect of $F_{m}$ is to smoothly stretch the cell $e_{\mu}^{m}$ across the expansion cell $\tilde{e}_{\mu}^{m+1}$ to the other side $\tilde{f}_{\mu}^{m+1}\left(\operatorname{cl}\left(\dot{\mathbf{I}}^{m+1}-\left\{\mathbf{I}^{m} \times 0\right\}\right)\right)$, while holding the rest of $K^{m}$ (including $\left.\operatorname{bdy}\left(e_{\mu}^{m}\right)\right)$ fixed.

Now, slide the attaching maps of $(m+1)$-cells $e_{\xi}^{m+1} \in K$ across the homotopy $F_{m}$ to obtain a homotopy $(m+1)$-move

$$
K(m)^{m+1}=K(m)^{m} \cup\left(\bigcup_{\xi \in \mathscr{S}_{m+1}} e_{\xi}^{m+1}\right) \stackrel{m+1}{\longrightarrow} K(m)^{m} \cup\left(\bigcup_{\xi \in \mathscr{I}_{m+1}} \bar{e}_{\xi}^{m+1}\right) .
$$


It is easy to check that the attaching maps $\bar{f}_{\xi}^{m+1}$ for the new cells $\bar{e}_{\xi}^{m+1}$ are model maps for the words $\bar{w}_{\xi}^{m+1}$, where $\bar{w}_{\xi}^{m+1}$ arises from $w_{\xi}^{m+1}$ by substituting via $\tilde{w}_{\mu}^{m+1}$.

Inductively, let $q$ satisfy $m+2 \leq q \leq n$. Suppose that a complex $K(q-2)$ and a homotopy $F_{q-2}: K^{q-2} \times \mathbf{I} \rightarrow K(q-2)$ have been defined such that there is a homotopy $(q-1)$-deformation

$$
K(q-2)^{q-2} \cup\left(\bigcup_{\alpha \in \mathcal{I}_{q-1}} e_{\alpha}^{q-1}\right) \stackrel{q-1}{\longrightarrow} K(q-2)^{q-2} \cup\left(\bigcup_{\alpha \in \mathcal{I}_{q-1}} \bar{e}_{\alpha}^{q-1}\right) .
$$

Let $\tilde{e}_{\alpha}^{q}$ be the expansion cell for the elementary homotopy $(q-1)$-move from $e_{\alpha}^{q-1}$ to $\bar{e}_{\alpha}^{q-1}\left(\alpha \in \mathscr{F}_{q-1}\right)$, and define

$$
K(q-1)=K(q-2) \cup\left(\bigcup_{\alpha \in \mathscr{J}_{q-1}}\left\{\bar{e}_{\alpha}^{q-1}, \tilde{e}_{\alpha}^{q}\right\}\right) .
$$

Let $F_{q-1}$ be the homotopy $F_{q-1}: K^{q-1} \times \mathbf{I} \rightarrow K(q-1)$ defined by

$$
F_{q-1}(x, t)= \begin{cases}F_{q-2}(x, t), & \text { if } x \in K^{q-2}, \\ \tilde{\phi}_{\alpha}^{q}\left(\left(\phi_{\alpha}^{q-1}\right)^{-1}(x), t\right), & \text { if } x \in e_{\alpha}^{q-1}, \alpha \in \mathcal{I}_{q-1} .\end{cases}
$$

(Note that no deformation retraction is interposed.) Then we may slide the attaching maps of $q$-cells $e_{\beta}^{q}$ across $F_{q-1}$ to obtain a homotopy $q$-deformation

$$
K(q-1)^{q}=K(q-1)^{q-1} \cup\left(\bigcup_{\beta \in \mathscr{J}_{q}} e_{\beta}^{q}\right) \stackrel{q}{\rightarrow} K(q-1)^{q-1} \cup\left(\bigcup_{\beta \in \mathscr{S}_{q}} \bar{e}_{\beta}^{q}\right) .
$$

The attaching maps $\bar{f}_{\beta}^{q}: \dot{\mathbf{I}}^{q} \rightarrow K(q-1)^{q-1}$ of new $q$-cells $\bar{e}_{\beta}^{q}$ are defined by $\bar{f}_{\beta}^{q}(y)=F_{q-1}\left(f_{\beta}^{q}(y), 1\right) \quad\left(y \in \dot{\mathbf{I}}^{q}\right)$, and each $\bar{f}_{\beta}^{q}$ is clearly a model map for $\bar{w}_{\beta}^{q}$, the word that arises from $w_{\beta}^{q}$ by substituting via $\tilde{w}_{\mu}^{m+1}$.

Define $K(n)=K(n-1)^{n-1} \cup\left(\bigcup_{\nu \in \mathcal{I}_{n}} \bar{e}_{\nu}^{n}\right)$, and let $\overline{\mathscr{W}}$ arise from $\mathscr{W}$ by substituting via $\tilde{w}_{\mu}^{m+1}$. Then there is a homotopy $n$-deformation

$$
\begin{aligned}
& K \underset{e, m+1}{\nearrow} K(m) \stackrel{m+2}{\nearrow} K(m+1) \nearrow^{m+3} \cdots \nearrow^{n} K(n-1) \stackrel{n}{\rightarrow} K(n) \\
& \searrow \bar{K}=\left(K^{m} \cup \bar{e}_{\mu}^{m}-e_{\mu}^{m}\right) \cup\left(\bigcup_{\xi \in \mathscr{I}_{m+1}} \bar{e}_{\xi}^{m+1}\right) \cup \cdots \cup\left(\bigcup_{\nu \in \mathcal{Y}_{n}} \bar{e}_{\nu}^{n}\right)
\end{aligned}
$$

where $\bar{K}$ is a geometric realization of $\overline{\mathscr{W}}$.

We now present two applications of geometric dragging.

Proposition (3.3.4) (Existence of model complexes). If a geometric realization $K$ exists for a word system $\mathscr{W}$ of order $n$, then $K$ homotopy $n$-deforms to a model complex $K(\mathscr{W})$ for $\mathscr{W}$.

Proof. Internally collapse a maximal tree (3.3.2) to obtain a complex $\widetilde{K}$ with only one vertex such that $K \stackrel{n}{\sim} \widetilde{K}$. By Proposition (3.2.3) and geometric 
dragging (3.3.3) we may deform $\widetilde{K}$ to a complex $K(n)$ whose $n$-cells are model cells. Inductively by the same reasoning, there is a homotopy deformation $K(m+1) \stackrel{n}{\cap} K(m) \quad(2 \leq m \leq n-1)$ to a complex $K(m)$ whose cells $\bar{e}_{\sigma}^{s}$ of dimension $s \geq m$ are model cells. Therefore, $K \stackrel{n}{\neg} K(2)$, where $K(2)$ is clearly a model complex for $\mathscr{W}$.

Proposition (3.3.5). If $K$ and $\bar{K}$ are geometric realizations of the same word system $\mathscr{W}$ of order $n$, then $K \stackrel{n}{\wedge} \bar{K}$.

Proof. By Proposition (3.3.4), we may assume that $K$ and $\bar{K}$ are model complexes without loss of generality.

Perform an elementary 1-expansion $K \nearrow_{e, q} K \cup\left\{\bar{e}^{0}, \tilde{e}^{1}\right\}$, where $\tilde{e}^{1}$ is a 1-cell joining the unique 0 -cells $e^{0} \in K$ and $\bar{e}^{0} \in \bar{K}$. Slide the (trivial) attaching maps of 1-cells $e_{\alpha}^{1} \in K$ along $\tilde{e}^{1}$ to obtain a homotopy 1-move

$$
(K)^{1} \cup\left\{\bar{e}^{0}, \tilde{e}^{1}\right\} \stackrel{1}{\longrightarrow}(\bar{K})^{1} \cup\left\{e^{0}, \tilde{e}^{1}\right\} .
$$

Then $K \cup\left\{\bar{e}^{0}, \tilde{e}^{1}\right\} \stackrel{n}{\overbrace{}^{\prime}} L \underset{e, 1}{\searrow} K(1)$ where $L$ is obtained by geometric dragging and $K(1)=L-\left\{e^{0}, \tilde{e}^{1}\right\}$. Observe that $K(1)$ is a model complex for $\mathscr{W}$ with $K(1)^{1}=(\bar{K})^{1}$.

Continue this process in each higher dimension. Since the attaching maps $f_{\mu}^{m}$ for $e_{\mu}^{m} \in K(1)$ and $\bar{f}_{\mu}^{m}$ for $\bar{e}_{\mu}^{m} \in \bar{K}$ are associated with the same word $w_{\mu}^{m} \in \mathscr{W}$, we see $f_{\mu}^{m} \simeq \bar{f}_{\mu}^{m}$. Thus, there is a homotopy $m$-deformation

$$
K(m-1)^{m} \stackrel{m}{\longrightarrow} K(m-1)^{m} \cup\left(\bigcup_{\mu} \bar{e}_{\mu}^{m}\right)-\left(\bigcup_{\mu} e_{\mu}^{m}\right) .
$$

Then $K(m-1) \stackrel{n}{\Gamma^{\prime}} K(m)$ by geometric dragging (3.3.3), where $K(m)$ satisfies $K(m)^{m}=\bar{K}^{m}$.

It follows by induction that $K$ homotopy $n$-deforms to the complex $\bar{K}=$ $K(n)$.

\section{Algebraic Form of the Main theorem}

4.1 Algebraic Equivalence Theorem. We will prove the following result, which establishes most of Theorem (6.1.1).

Theorem (4.1.1) (Algebraic Equivalence Theorem). Let $\mathscr{W}$ and $\mathscr{W}^{\prime}$ be word systems of order $n$. Then the following are equivalent:

(1) $\mathscr{W}$ is $P_{n}$-equivalent to $\mathscr{W}^{\prime}$.

(2) $\mathscr{W}$ is fully $P_{n}$-equivalent to $\mathscr{W}^{\prime}$.

(3) $\mathscr{W}$ is $D_{n+1}$-equivalent to $\mathscr{W}^{\prime}$.

In this section we will show the implication $(3) \Rightarrow(1)$, which is the central idea behind the result. The remainder of the proof involves technical arguments that are summarized in the next section. 
Theorem (4.1.2). If $\mathscr{W}$ is $D_{n+1}$-equivalent to $\mathscr{W}^{\prime}$ then $\mathscr{W}$ is $P_{n}$-equivalent to $\mathscr{W}^{\prime}$.

Proof. Let $\mathscr{W}=\mathscr{W}_{0}, \mathscr{W}_{1}, \ldots, \mathscr{W}_{R}=\mathscr{W}^{\prime}$ be a $D_{n+1}$-sequence from $\mathscr{W}$ to $\mathscr{W}^{\prime}$. We will use the notation $w_{\mu}^{m}\left(\mathscr{W}_{r}\right)$ when necessary to specify which word system $\mathscr{W}_{r}$ contains a given word $w_{\mu}^{m}$.

Since $\mathscr{W}_{0}$ is a word system of order $n$, every $(n+1)$-word that occurs in a word system $\mathscr{W}_{r}(1 \leq r \leq R-1)$ must arise from an $(n+1)$-expansion prior to the move $\left[\mathscr{W}_{r}, \mathscr{W}_{r+1}\right]$ in the $D_{n+1}$-sequence. Hence, that $(n+1)$-word must have been introduced together with a new $n$-word for its expansion face. For bookkeeping purposes, we will choose nonzero integers that satisfy the following conditions to be the indices of $n$-words and $(n+1)$-words of $\mathscr{W}_{r}$.

Indexing conditions on $\mathscr{W}_{r}$. (a) Positive integer indices, indicating the order of appearance in the $D_{n+1}$-sequence $\mathscr{W}=\mathscr{W}_{0}, \mathscr{W}_{1}, \ldots, \mathscr{W}_{r}$, are assigned to any $(n+1)$-words $w_{+i}^{n+1}$ that are present in $\mathscr{W}_{r}$, and the same indices are assigned to the corresponding expansion face $n$-words $w_{+i}^{n} \in \mathscr{W}_{r}$. (b) Negative integer indices are assigned to all $n$-words of $\mathscr{W}_{r}$ that are not included in case (a).

If $\mathscr{W}_{r}$ satisfies the indexing conditions, define the word system

$$
\mathscr{V}_{r}=\mathscr{W}_{r}-\bigcup_{+i>0}\left\{w_{+i}^{n}, w_{+i}^{n+1}\right\}
$$

$\mathscr{V}_{r}$ is the result of collapsing all $(n+1)$-words $w_{+i}^{n+1} \in \mathscr{W}_{r}$ in reverse order of appearance through their expansion face $n$-words $w_{+i}^{n} \in \mathscr{W}_{r}$. Choose negative integer indices for all $n$-words in $\mathscr{W}=\mathscr{W}_{0}$.

Suppose that $\mathscr{W}_{r}$ satisfies the indexing conditions, and assume that a $P_{n}$ sequence exists from $\mathscr{V}_{0}=\mathscr{W}$ to the subsystem $\mathscr{V}_{r}$ of $\mathscr{W}_{r}$. We shall find indexing on $\mathscr{W}_{r+1}$ satisfying the indexing conditions such that $\mathscr{\mathscr { V }}_{r}$ is $P_{n}$-equivalent to $\mathscr{V}_{r+1}$. We consider each case of the $D_{n+1}$-move $\left[\mathscr{W}_{r}, \mathscr{W}_{r+1}\right]$.

Case 1. Inversion, $\left(w_{\mu}^{m}\right)^{-1}$. Choose the same indexing for $\mathscr{W}_{r+1}$ as for $\mathscr{W}_{r}$ in order to preserve the indexing conditions. If $m \leq n$, then $\mathscr{V}_{r+1}$ results from $\mathscr{V}_{r}$ by the same inversion $\left(w_{\mu}^{m}\right)^{-1}$. If $m=n+1$ then $\mathscr{V}_{r}=\mathscr{V}_{r+1}$.

Case 2. Elementary m-deformation, $m \leq n$. Select indexing for $\mathscr{W}_{r+1}$ that is consistent with the indexing for $\mathscr{W}_{r}$. In the case of an $n$-expansion, assign a negative integer index to the expansion $n$-word. Then $\mathscr{V}_{r+1}$ is obtained from $\mathscr{V}_{r}$ by the same elementary $m$-deformation $\left[\mathscr{W}_{r}, \mathscr{W}_{r+1}\right]$.

Case 3. Elementary $(n+1)$-expansion. Assign the index $+r$ to both new words $w_{+r}^{n}$ and $w_{+r}^{n+1}$. For other words, choose the same indexing for $\mathscr{W}_{r+1}$ as for $\mathscr{W}_{r}$. The indexing conditions are satisfied and we have $\mathscr{V}_{r+1}=\mathscr{V}_{r}$.

Case 4. Elementary $(n+1)$-collapse, with $w_{+k}^{n+1}$ and $w_{\beta}^{n}$ removed. If the collapse face $n$-index $\beta$ is positive, then we must have $\beta=+k$. This is because $w_{\beta}^{n+1}$ is present in $\mathscr{W}_{r}$, and thus $w_{\beta}^{n}$ cannot be a free face of $w_{+k}^{n+1}$ unless $\beta=+k$. Choose the same indexing in $\mathscr{W}_{r+1}$ as in $\mathscr{W}_{r}$ and observe that $\mathscr{V}_{r+1}=\mathscr{V}_{r}$.

Suppose that the collapse face $n$-index $\beta$ is negative, $\beta=-j$. Then the collapse face index $-j \in \mathscr{I}_{n}$ differs from the expansion face index $+k \in \mathscr{I}_{n}$ for $w_{+k}^{n+1}\left(\mathscr{W}_{r}\right)$. Assign indices in $\mathscr{W}_{r+1}$ consistent with the indexing in $\mathscr{W}_{r}$, except reindex $w_{+k}^{n}\left(\mathscr{W}_{r}\right)$ as $w_{-j}^{n}\left(\mathscr{W}_{r+1}\right)$. Then $w_{-j}^{n}\left(\mathscr{W}_{r+1}\right)$ is the expansion face and $w_{-j}^{n}\left(\mathscr{W}_{r}\right)$ is the collapse face for $w_{+k}^{n+1}$, and we seek a $P_{n}$-sequence from $\mathscr{V}_{r}$ to $\mathscr{V}_{r+1}=\mathscr{V}_{r} \cup\left\{w_{-j}^{n}\left(\mathscr{W}_{r+1}\right)\right\}-\left\{w_{-j}^{n}\left(\mathscr{W}_{r}\right)\right\}$. 
Solve $w_{+k}^{n+1} \in \mathscr{W}_{r}$ for $(1,+k)$ in the sense of Subsection 3.3 to obtain a word $\hat{w}_{+k}^{n+1} \doteq(1,+k)$. Then $\hat{w}_{+k}^{n+1}$ mentions the index $-j$ exactly once, and any positive index $+i$ occurring in $\hat{w}_{+k}^{n+1}$ satisfies $+i<+k$ by the indexing criteria. Let $+i_{0}$ be the highest such index $+i$. Then we may solve $w_{+i_{0}}^{n+1}$ for $\left(1,+i_{0}\right)$ to obtain $\hat{w}_{+i_{0}}^{n+1} \doteq\left(1,+i_{0}\right)$, where again the only positive integer indices $+h$ occurring in $\hat{w}_{+i_{0}}^{n+1}$ satisfy $+h<+i_{0}$. Now, substitute $\hat{w}_{+i_{0}}^{n+1}$ for $\left(1,+i_{0}\right)$ in $\hat{w}_{+k}^{n+1}$, yielding a word $W_{1} \doteq(1,+k)$ such that all positive integers $+a$ mentioned in $W_{1}$ satisfy $+a<+i_{0}$. Furthermore, $W_{1}$ still mentions $-j$ exactly once, since that index cannot be mentioned in $\hat{w}_{+i_{0}}^{n+1}$ as $w_{-j}^{n}$ is a free face of $w_{+k}^{n+1}$.

Continue this process, defining $(n+1)$-words $W_{2} \doteq W_{3} \doteq \cdots$ with decreasing highest positive integer $n$-index. The process must stop in a word $W_{l}$ that contains no positive integer $n$-indices. We have $W_{l} \doteq(1,+k)$, and $W_{l}$ mentions the index $-j$ associated with the word system $\mathscr{W}_{r}$ exactly once.

Now, write $W_{l}$ in the form

$$
\begin{aligned}
W_{l}= & \varepsilon_{1}\left(g_{1},-p_{1}\right)+\cdots+\varepsilon_{q-1}\left(g_{q-1},-p_{q-1}\right)+\varepsilon_{q}\left(g_{q},-j\right) \\
& +\varepsilon_{q+1}\left(g_{q+1},-p_{q+1}\right)+\cdots+\varepsilon_{Q}\left(g_{Q},-p_{Q}\right) .
\end{aligned}
$$

When $n \leq 3$, we write

$$
W_{l}=\left\{\begin{array}{rr}
\left(g_{1},-p_{1}\right)^{\varepsilon_{1}} \cdots\left(g_{q-1},-p_{q-1}\right)^{\varepsilon_{q-1}}\left(g_{q},-j\right)^{\varepsilon_{q}}\left(g_{q+1},-p_{q+1}\right)^{\varepsilon_{q+1}} \\
\cdots\left(g_{Q},-p_{Q}\right)^{\varepsilon_{Q}} & \text { when } n=3, \\
\left(-p_{1}\right)^{\varepsilon_{1}} \cdots\left(-p_{q-1}\right)^{\varepsilon_{q-1}}(-j)^{\varepsilon_{q}}\left(-p_{q+1}\right)^{\varepsilon_{q+1}} \cdots\left(-p_{Q}\right)^{\varepsilon_{q}} & \text { when } n=2 .
\end{array}\right.
$$

Then we may use the form of $W_{l}$ as a guide to find a sequence of $P_{n}$-moves from $\mathscr{V}_{r}$ to $\mathscr{V}_{r+1}$, as follows.

1. Conjugate $w_{-j}^{n} \in \mathscr{V}_{r}$ by the group element $g_{q}$, yielding $g_{q} \cdot w_{-j}^{n}$ (conjugation is omitted when $n=2$ ).

2. Invert $g_{q} \cdot w_{-j}^{n}$ if necessary to obtain $\varepsilon_{q} g_{q} \cdot w_{-j}^{n}$ (or $g_{q} \cdot\left(w_{-j}^{n}\right)^{\varepsilon_{q}}$ when $n=3$, or $\left(w_{-j}^{n}\right)^{\varepsilon_{q}}$ when $\left.n=2\right)$.

3. Conjugate and invert $w_{-p_{q+1}}^{n}$ to obtain $\varepsilon_{q+1} g_{q+1} \cdot w_{-p_{q+1}}^{n}$.

4. Perform an $n$-product equivalence yielding $\varepsilon_{q} g_{q} \cdot w_{-j}^{n}+\varepsilon_{q+1} g_{q+1} \cdot w_{-p_{q+1}}^{n}$ (or an analogous form if $n=2$ or 3 ).

5. Conjugate and invert $\varepsilon_{q+1} g_{q+1} \cdot w_{-p_{q+1}}^{n}$ back to $w_{-p_{q+1}}^{n}$.

6. Conjugate and invert $w_{-p_{q+2}}^{n}$ to obtain $\varepsilon_{q+2} g_{q+2} \cdot w_{-p_{q+2}}^{n}$.

7. Perform an $n$-product equivalence yielding

$$
\varepsilon_{q} g_{q} \cdot w_{-j}^{n}+\varepsilon_{q+1} g_{q+1} \cdot w_{-p_{q+1}}^{n}+\varepsilon_{q+2} g_{q+2} \cdot w_{-p_{q+2}}^{n} .
$$

8. Continue with similar conjugations, inversions and $n$-product equivalences.

Then $\mathscr{V}_{r}$ is $P_{n}$-equivalent to the word system $\overline{\mathscr{V}}_{r}=\mathscr{V}_{r} \cup\left\{\bar{w}^{n}\right\}-\left\{w_{-j}^{n}\right\}$, where the $n$-word $\bar{w}^{n}$ has the form

$$
\begin{aligned}
\bar{w}^{n}= & \varepsilon_{1} g_{1} \cdot w_{-p_{1}}^{n}+\cdots+\varepsilon_{q-1} g_{q-1} \cdot w_{-p_{q-1}}^{n}+\varepsilon_{q} g_{q} \cdot\left(w_{-j}^{n}\right) \\
& +\varepsilon_{q+1} g_{q+1} \cdot w_{-p_{q+1}}^{n}+\cdots+\varepsilon_{Q} g_{Q} \cdot w_{-p_{Q}}^{n}
\end{aligned}
$$

(or an analogous form if $n=2$ or 3). Clearly, $\left\langle\bar{w}^{n}\right\rangle=d_{n+1}\left(\left\langle W_{l}\right\rangle\right)$. Since $W_{l} \doteq w_{+k}^{n}$ and $\left\langle w_{+k}^{n}\right\rangle=d_{n+1}(\langle 1,+k\rangle)$, we conclude that $\bar{w}^{n}$ represents the 
same element in $M_{n}\left(\mathscr{I}_{n-1}\right)$ as the word $w_{+k}^{n}\left(\mathscr{W}_{r}\right)=w_{-j}^{n}\left(\mathscr{W}_{r+1}\right)$. Thus, a wordrepresentative equivalence transforms $\overline{\mathscr{V}}_{r}$ into $\mathscr{V}_{r+1}$. Hence there exists a $P_{n}$-sequence from $\mathscr{V}_{r}$ to $\mathscr{V}_{r+1}$.

We conclude by induction that there is a $P_{n}$-sequence from $\mathscr{V}_{0}=\mathscr{W}$ to $\mathscr{V}_{R}=\mathscr{W}^{\prime}$.

5.1 Remainder of the proof of the Algebraic Equivalence Theorem. Since $P_{n}$ equivalence implies full $P_{n}$-equivalence, Theorem (4.1.1) will follow if we can show that fully $P_{n}$-equivalent word systems are $D_{n+1}$-equivalent. We will show this using an algebraic counterpart of Proposition (3.3.3), called algebraic dragging (5.1.2). The easiest way to establish algebraic dragging is by converting to a geometric problem and applying (3.3.3). Unfortunately, this cannot be done in general for higher-order word systems, as Example (2.2.3) shows. We will take this geometric approach in low dimensions in order to avoid some unpleasant noncommutative algebra.

Lemma (5.1.1). Let $\mathscr{W} \nearrow \nearrow_{e, m} \mathscr{W}^{\prime}=\mathscr{W} \cup\left\{w_{\lambda}^{m-1}, w_{\mu}^{m}\right\}$ be an elementary $m$ expansion of word systems. If $K=K(\mathscr{W})$ is a model complex for $\mathscr{W}$, then $K \nearrow_{e, m} K^{\prime}$ where $K^{\prime}=K\left(\mathscr{W}^{\prime}\right)$ is a model complex for $\mathscr{W}^{\prime}$.

Proof. It suffices to show that model cells $e_{\lambda}^{m-1}$ for $w_{\lambda}^{m-1}$ and $e_{\mu}^{m}$ for $w_{\mu}^{m}$ exist. This is straightforward when $m=2$.

Suppose $m \geq 3$. Solve the word $w_{\mu}^{m}$ for $(1, \lambda)$ in the sense of Subsection 3.3, yielding $\hat{w}_{\mu}^{m} \doteq(1, \lambda)$. Construct spaces $X \subset N \subset S^{m-1}$ and a map $\widetilde{F}: N \rightarrow K^{m-1}$ such that $\hat{w}_{\mu}^{m}$ is associated with $\widetilde{F}, \hat{w}_{\mu}^{m} \sim \widetilde{F}$, as in the proof of Proposition (3.2.3). The neighborhood $N$ is a ball, so $\partial \widetilde{F}:(\operatorname{bdy}(N), *) \rightarrow$ $\left(K^{m-2}, *\right)$ represents an element $[\partial \widetilde{F}] \in \pi_{m-2}\left(K^{m-2}, K^{m-3}\right)$. Trivially, $w_{\lambda}^{m-1}$ $\sim \partial \widetilde{F}$. In fact, $\partial \widetilde{F}$ actually represents an element in the absolute homotopy group $\pi_{m-2}\left(K^{m-2}\right)$, so by the long exact sequence in homotopy, $\beta([\partial \widetilde{F}]) \in$ $\pi_{m-3}\left(K^{m-3}\right)$ is the trivial element. Therefore, we may apply (3.2.3) to obtain a homotopy $H: \partial \widetilde{F} \simeq f_{\lambda}^{m-1}$ to a model map $f_{\lambda}^{m-1}$ for $w_{\lambda}^{m-1}$. Let $e_{\lambda}^{m-1}$ be a new $(m-1)$-cell attached to $K^{m-2}$ by $f_{\lambda}^{m-1}$.

Let $\overline{\phi_{\lambda}^{m-1}}$ be the characteristic map for $e_{\lambda}^{m-1}$ with orientation reversed. Then we may paste together the map $\tilde{F}$, the homotopy $H$ and a copy of $\overline{\phi_{\lambda}^{m-1}}$ to obtain a model map $F_{1}: S^{m-1} \rightarrow K^{m-1}$ for the word $w_{1}=-(1, \lambda)+\hat{w}_{\mu}^{m}$ (making trivial choices of basepoint and connecting path for $e_{\lambda}^{m-1}$ ). Modify $F_{1}$ on a small neighborhood of the basepoint of $S^{m-1}$, yielding a map $F_{2}: S^{m-1} \rightarrow$ $K^{m-1}$ that is a model map for the word $w_{2}=-(g, \lambda)+g \cdot \hat{w}^{m}$, where $g$ is the coefficient of $\lambda$ in the expansion word $w_{\mu}^{m} \in \mathscr{W}$. Clearly, $w_{2}$ is a cyclic permutation of either $w_{\mu}^{m}$ or $-w_{\mu}^{m}$. Therefore, either $F_{2}$ or $\overline{F_{2}}$ is a model map $f_{\mu}^{m}: S^{m-1} \rightarrow K^{m-1}$ for the word $w_{\mu}^{m}$, by Proposition (3.2.2). Attach a new cell $e_{\mu}^{m}$ to $K^{m-1}$ via $f_{\mu}^{m}$. Then we have constructed model cells $e_{\lambda}^{m-1}$ and $e_{\mu}^{m}$. 
Proposition (5.1.2) (Algebraic dragging technique). Let $\mathscr{W}$ be a word system of order $n$, and let $w_{\mu}^{m} \in \mathscr{W}$. Suppose that $\bar{w}_{\bar{\mu}}^{m}$ and $\tilde{w}_{\tilde{\mu}}^{m+1}$ are words representing elements in $M_{m}(\mathscr{W})$ and $M_{m+1}(\mathscr{W})$ such that

(a) $\bar{w}_{\bar{\mu}}^{m}$ has a new m-index $\bar{\mu} \notin \mathscr{I}_{m}(\mathscr{W})$ and satisfies $d\left\langle\left(\bar{w}_{\bar{\mu}}^{m}\right)\right\rangle=1$, and

(b) $\tilde{w}_{\tilde{\mu}}^{m+1}$ has a new $(m+1)$-index $\tilde{\mu} \notin \mathscr{I}_{m+1}(\mathscr{W})$, satisfies $d\left\langle\tilde{w}_{\tilde{\mu}}^{m+1}\right\rangle=1$ and mentions the $m$-indices $\mu$ and $\bar{\mu}$ exactly once each.

Let $\overline{\mathscr{W}}$ arise from $\mathscr{W}$ by substituting via $\tilde{w}_{\tilde{\mu}}^{m+1}$, solving for $(1, \mu)$ (or solving for $\mu$ in the case $n=2$ ).

Then $\stackrel{\mathscr{W}}{m+1}_{m+1}^{n+1} \overline{\mathscr{W}}$.

We first establish the following special case.

Lemma (5.1.3). Proposition (5.1.2) holds if $\mathscr{W}$ has a geometric realization. Furthermore, in that case we may rearrange the $D_{n+1}$-sequence so that all expansions occur first, i.e., the deformation has the form $\mathscr{W}_{m+1}^{n+1} \mathscr{W}^{\prime} \searrow_{m+1}^{n+1} \overline{\mathscr{W}}$.

Proof of lemma. By Proposition (3.3.4) a model complex $K=K(\mathscr{W})$ exists for $\mathscr{W}$. Let $\bar{e}_{\bar{\mu}}^{m}$ and $\tilde{e}_{\tilde{\mu}}^{m+1}$ be model cells reflecting the expansion $\overline{\mathscr{W}} \nearrow_{e, m+1}$ $\mathscr{W} \cup\left\{\bar{w}_{\bar{\mu}}^{m}, \tilde{w}_{\dot{\mu}}^{m+1}\right\}$ as in Lemma (5.1.1). Examining the proof, we see that there is a homotopy $m$-move $K^{m} \underset{e, m}{\longrightarrow} K^{m} \cup \bar{e}_{\bar{\mu}}^{m}-e_{\mu}^{m}$ and that the elementary $(m+1)$-expansion associated with this homotopy move involves the cells $\bar{e}_{\bar{\mu}}^{m}$ and $\tilde{e}_{\tilde{\mu}}^{m+1}$. Apply Proposition (3.3.3) to obtain a homotopy $n$-deformation $K \overbrace{m}^{n} \bar{K}$ where $\bar{K}=K(\overline{\mathscr{W}})$ is a model complex for $\overline{\mathscr{W}}$. Then there is an associated formal $(n+1)$-deformation $K \underset{m+1}{n+1} \bar{K}$.

That deformation may be rearranged so that all expansions come first

$$
K \underset{m+1}{n+1} K^{\prime} \underset{m+1}{n+1} \bar{K}
$$

by a standard argument. An examination of the proof of Proposition (3.3.3) shows that $K^{\prime}$ is a model complex for a word system $\mathscr{W}^{\prime}$. Now apply the idea

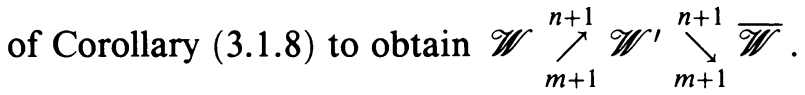

Proof of Proposition (5.1.2). Let $w_{\rho}^{r}$ denote a typical word in $\mathscr{W}$. After reindexing, $\overline{\mathscr{W}}$ consists of:

$w_{\rho}^{r}(r<m)$;

$w_{\zeta}^{m}(\zeta \neq \mu)$ and $\bar{w}_{\bar{\mu}}^{m} ;$ and

$\bar{w}_{\bar{\rho}}^{r}(r>m)$, where $\bar{w}_{\bar{\rho}}^{r}$ arises from $w_{\rho}^{r}$ by substituting for $\mu$ via $\tilde{w}_{\tilde{\mu}}^{m+1}$.

It is enough to define expansion words $\tilde{w}_{\tilde{\rho}}^{r+1}\left(\rho \in \mathscr{T}_{r}, m+1 \leq r \leq n\right)$ satisfying the conditions

(i) $\tilde{w}_{\tilde{\rho}}^{r+1}$ mentions the indices $\rho$ and $\bar{\rho}$ exactly once each, and

(ii) $d_{r+1}\left(\left\langle\tilde{w}_{\tilde{\rho}}^{r+1}\right\rangle\right)$ is trivial, 
for then we may deform $\mathscr{W}$ to $\overline{\mathscr{W}}$ as in (5.1.4).

$$
\begin{gathered}
\mathscr{W} \nearrow \mathscr{W} \cup\left\{\bar{w}_{\bar{\mu}}^{m}, \tilde{w}_{\tilde{\mu}}^{m+1}\right\} \cup\left(\bigcup_{\xi \in \mathcal{I}_{m+1}}\left\{\bar{w}_{\bar{\xi}}^{m+1}, \tilde{w}_{\tilde{\xi}}^{m+2}\right\}\right) \\
\cup \cdots \cup\left(\bigcup_{\nu \in \mathscr{I}_{n}}\left\{\bar{w}_{\bar{\nu}}^{n}, \tilde{w}_{\tilde{\nu}}^{n+1}\right\}\right) \searrow \overline{\mathscr{W}} .
\end{gathered}
$$

We argue by induction on $r$. As the initial case we claim that expansion words $\tilde{w}_{\rho}^{r+1}$ exist for $r=\max (m+1,4)$. When $m<4$, consider the 4-section $\mathscr{W}^{4}=\left\{w_{\pi}^{p} \in \mathscr{W} \mid p \leq 4\right\}$. $\mathscr{W}^{4}$ has a geometric realization by Proposition (2.2.4), so we may apply the lemma to obtain a 5-expansion $\mathscr{W}^{4} \nearrow_{m+1}^{5} \mathscr{W}^{\prime}$, where

$$
\mathscr{W}^{\prime}=\mathscr{W}^{4} \cup\left\{\bar{w}_{\bar{\mu}}^{m}, \tilde{w}_{\tilde{\mu}}^{m+1}\right\} \cup\left(\bigcup_{\xi \in \mathcal{I}_{m+1}}\left\{\bar{w}_{\bar{\xi}}^{m+1}, \tilde{w}_{\tilde{\xi}}^{m+2}\right\}\right) \cup \cdots \cup\left(\bigcup_{\delta \in \mathscr{I}_{4}}\left\{\bar{w}_{\bar{\delta}}^{4}, \tilde{w}_{\tilde{\delta}}^{5}\right\}\right) .
$$

An examination of the proof of Proposition (3.3.3) and the lemma shows

$$
\tilde{w}_{\tilde{\delta}}^{5}=(1, \delta)-(1, \bar{\delta})-w_{\delta}^{5}\left(\widetilde{\mathscr{J}_{3}}\right)
$$

Here we write $w_{\lambda}^{l+1}\left(\widetilde{\mathscr{J}}_{l-1}\right)$ to denote the result of replacing each $(l-1)$-index $\kappa$ in $w_{\lambda}^{l}$ by the $l$-index $\tilde{\kappa}$. Then $\tilde{w}_{\tilde{\delta}}^{5}$ satisfies condition (i), and the boundary condition (ii) holds by the lemma.

When $m \geq 4$, we may write $\tilde{w}_{\tilde{\mu}}^{m+1}$ in the form $\tilde{w}_{\tilde{\mu}}^{m+1}=\varepsilon(g, \mu)+\varepsilon g \cdot \hat{w}_{\tilde{\mu}}^{m+1}$ where $\hat{w}_{\tilde{\mu}}^{m+1}$ arises from $\tilde{w}_{\tilde{\mu}}^{m+1}$ by solving for $(1, \mu)$. If $w_{\xi}^{m+1}$ is any $(m+1)=$ word, it can be written in the form $w_{\xi}^{m+1}=A_{0}+\varepsilon_{1}\left(g_{1}, \mu\right)+A_{1}+\cdots+\varepsilon_{Q}\left(g_{Q}, \mu\right)+$ $A_{Q}$. Define

$$
\tilde{w}_{\tilde{\xi}}^{m+2}=(1, \xi)-(1, \bar{\xi})-\varepsilon g^{-1} \cdot\left(\varepsilon_{1}\left(g_{1}, \tilde{\mu}\right)+\cdots+\varepsilon_{Q}\left(g_{Q}, \tilde{\mu}\right)\right) .
$$

This word satisfies (i), and (ii) follows by the same reasoning as in the inductive step below.

Assume that words $\tilde{w}_{\tilde{\rho}}^{r+1}\left(\rho \in \mathscr{I}_{r}\right)$ have been defined, where

$$
\max (m+1,4) \leq r<n .
$$

Then define the words $\left(\sigma \in \mathscr{F}_{r+1}\right)$

$$
\tilde{w}_{\tilde{\sigma}}^{r+2}=(1, \sigma)-(1, \bar{\sigma})-w_{\sigma}^{r+2}\left(\widetilde{\mathscr{J}_{r}}\right)
$$

representing an element in the module $M_{r+2}\left(\mathscr{I}_{r+1} \cup \overline{\mathscr{I}}_{r+1} \cup \widetilde{\mathscr{I}}_{r}\right)$. Using commutativity and the definition of substitution, we see that $d_{r+2}\left(\left\langle w_{\sigma}^{r+2}\left(\widetilde{\mathscr{J}}_{r}\right)\right\rangle\right)$ is just the word needed to convert $w_{\sigma}^{r+1}$ to $\bar{w}_{\bar{\sigma}}^{r+1}$ in the commutative module $M_{r+1}\left(\mathscr{I}_{r} \cup \overline{\mathscr{I}}_{r} \cup \widetilde{\mathscr{I}}_{r-1}\right)$, so $d_{r+2}\left(\left\langle\tilde{w}_{\tilde{\sigma}}^{r+2}\right\rangle\right)$ is trivial. Thus $\tilde{w}_{\tilde{\sigma}}^{r+2}$ satisfies (i) and (ii). This completes the induction.

Remark (5.1.5). The $D_{n+1}$-sequence $\mathscr{W}_{m+1}^{n+1} \overline{\mathscr{W}}$ that results from algebraic dragging (Proposition (5.1.2)) may be assumed transient. 
To see this, we examine the proof and note that $(n+1)$-words can arise in the proof of Proposition (5.1.2) only in the following instances.

1. If $\mathscr{W}$ has a geometric realization, then $(n+1)$-words arise in the proof of Lemma (5.1.3). Those words correspond to expansion $(n+1)$-cells associated with a homotopy $n$-deformation $K \overbrace{m}^{n} \bar{K}$ of model complexes $K, \bar{K}$ of dimension $n$. Lemma (1.2.3) implies that the associated formal $(n+1)$ deformation $K^{n+1} \wedge$ is transient, so a transient $D_{n+1}$-sequence exists in that case.

2. In the proof of Proposition (5.1.2) for the general case, the deformation (5.1.4) can clearly be rearranged into a transient deformation.

Proposition (5.1.6). Suppose that $\mathscr{W}=\mathscr{W}_{0}, \ldots, \mathscr{W}_{Q}=\overline{\mathscr{W}}$ is a full $P_{n}$-sequence of word systems of order $n$. Then there is a $D_{n+1}$-sequence from $\mathscr{W}$ to $\overline{\mathscr{W}}$.

Proof. We argue by induction on $q(0 \leq q \leq Q)$. Suppose that a $D_{n+1^{-}}$ sequence exists from $\mathscr{W}_{0}$ to $\mathscr{W}_{q}$. If the full $P_{n}$-move $\left[\mathscr{W}_{q}, \mathscr{W}_{q+1}\right]$ is an inversion, elementary $m$-expansion or elementary $m$-collapse $(m \leq n)$, then we can immediately extend the $D_{n+1}$-sequence to $\mathscr{W}_{q+1}$.

Now consider the other full $P_{n}$-moves. Suppose that $\bar{w}_{\mu}^{m}=\alpha \cdot w_{\mu}^{m}$ is an $m$ conjugation. Then $\mathscr{W}_{q} \nearrow_{e, m+1} \mathscr{W}_{q} \cup\left\{\bar{w}_{\mu}^{m}, \tilde{w}_{\mu}^{m+1}\right\}$, where $\tilde{w}_{\mu}^{m+1}=(1, \bar{\mu})-(\alpha, \mu)$ (or $\tilde{w}_{\mu}^{3}=\bar{\mu} \alpha \mu^{-1} \alpha^{-1}$ when $m=2$ ). Perform algebraic dragging (5.1.2) to obtain a $D_{n+1}$-sequence of word systems $\mathscr{W}_{q} \cup\left\{\bar{w}_{\mu}^{m}, \tilde{w}_{\mu}^{m+1}\right\} \stackrel{n+1}{\wedge} \overline{\mathscr{W}}_{q}$. It is clear that $\overline{\mathscr{W}}_{q}$ satisfies $\overline{\mathscr{W}}_{q}=\mathscr{W}_{q+1} \cup\left\{w_{\mu}^{m}, \tilde{w}_{\mu}^{m+1}\right\}$, so we may collapse $\overline{\mathscr{W}}_{q} \underset{e, m}{\searrow} \mathscr{W}_{q+1}$.

A similar argument applies for the remaining full $P_{n}$-moves. If $w_{\mu}^{m} \sim \bar{w}_{\bar{\mu}}^{m}$ is a word-representative equivalence, then choose $\tilde{w}_{\mu}^{m+1}=(1, \bar{\mu})-(1, \mu)$ (or $\bar{\mu} \mu^{-1}$ when $\left.m=2\right)$. For $m$-product equivalence, $\bar{\mu}=\mu \tau$, use $\tilde{w}_{\mu}^{m+1}=$ $(1, \bar{\mu})-(1, \tau)-(1, \mu)$ (or $\tilde{w}_{\mu}^{3}=\bar{\mu} \tau^{-1} \mu^{-1}$ when $\left.m=2\right)$. Then we have obtained a $D_{n+1}$-sequence from $\mathscr{W}_{q}$ to $\mathscr{W}_{q+1}$ for every full $P_{n}$-move $\left[\mathscr{W}_{q}, \mathscr{W}_{q+1}\right]$.

Remark (5.1.7). Observe that the $D_{n+1}$-sequence of Proposition (5.1.6) may be assumed transient.

This holds because $(n+1)$-words $w^{n+1}$ that occur in the proof of that proposition satisfy one of the following conditions.

1. $w^{n+1}=\tilde{w}_{\mu}^{m+1}$ where $m=n$. In this case, no algebraic dragging takes place, and the sequence $\mathscr{W}_{q} \nearrow_{e, m+1} \mathscr{W}_{q} \cup\left\{\bar{w}_{\mu}^{m}, \tilde{w}_{\mu}^{m+1}\right\} \underset{e, m}{\searrow} \mathscr{W}_{q+1}$ is transient.

2. $w^{n+1}$ arises during algebraic dragging. Remark (5.1.5) shows that $\mathscr{W}_{q}^{n+1} \mathscr{W}_{q+1}$ may be assumed transient.

Proof of Theorem (4.1.1). Theorem (4.1.2) establishes $\left(D_{n+1}\right.$-equivalence $\Rightarrow P_{n}$ equivalence), and the implication $\left(P_{n}\right.$-equivalence $\Rightarrow$ full $P_{n}$-equivalence) is trivial. The implication (full $P_{n}$-equivalence $\Rightarrow D_{n+1}$-equivalence) follows from Proposition (5.1.6). 


\section{MAIN THEOREM}

6.1 The Formal Equivalence Theorem. We are now ready to prove the following.

Theorem (6.1.1) (Formal Equivalence Theorem). Suppose that $K$ and $K^{\prime}$ are $C W$-complexes with homotopy readings $\mathscr{W}$ and $\mathscr{W}^{\prime}$, respectively.

Then the following are equivalent:

(a) $\mathscr{W}$ and $\mathscr{W}^{\prime}$ are $P_{n}$-equivalent.

(b) $\mathscr{W}$ and $\mathscr{W}^{\prime}$ are fully $P_{n}$-equivalent.

(c) $\mathscr{W}$ and $\mathscr{W}^{\prime}$ are $D_{n+1}$-equivalent.

(d) $K$ formally $(n+1)$-deforms to $K^{\prime}$.

Proof. Theorem (4.1.1) shows (a) $\Leftrightarrow$ (b) $\Leftrightarrow$ (c). Furthermore, suppose that $\mathscr{W}$ is $D_{n+1}$-equivalent to $\mathscr{W}^{\prime}$. Since $K$ is a geometric realization of $\mathscr{W}$, there is a model complex $K(\mathscr{W})$ such that $K \stackrel{n}{\Gamma^{\prime}} K(\mathscr{W})$ by Proposition (3.3.4). It follows that $K^{n+1} \wedge(\mathscr{W})$ by Lemma (1.2.3). Then we can emulate every $D_{n+1}$-move from $\mathscr{W}$ to $\mathscr{W}^{\prime}$ by a corresponding move between model complexes, using Lemma (5.1.1) for expansions, the definition of a model complex for collapses and orientation reversal of characteristic maps for inversion. Then $K(\mathscr{W})^{n+1} \wedge K\left(\mathscr{W}^{\prime}\right)$ where $K\left(\mathscr{W}^{\prime}\right)$ is a model complex for $\mathscr{W}^{\prime}$. Finally, $K\left(\mathscr{W}^{\prime}\right) \stackrel{n+1}{\wedge} K^{\prime}$ using Proposition (3.3.5), so there is a formal $(n+1)$-deformation from $K$ to $K^{\prime}$. This shows (c) $\Rightarrow(\mathrm{d})$.

It suffices to show (d) $\Rightarrow$ (b). Let $K=K_{0}, K_{1}, \ldots, K_{Q}=K^{\prime}$ be a formal deformation of $n$-complexes $K$ and $K^{\prime}$, and suppose that $\mathscr{W}$ is a homotopy reading of $K$. Then $\mathscr{W}$ is fully $P_{n}$-equivalent to a word system $\mathscr{W}_{0}$ that is a homotopy reading of $K$ consisting of geometric words, by Propositions (3.1.7) and (2.3.8). We now show that $\mathscr{W}_{0}$ is fully $P_{n}$-equivalent to a homotopy reading $\mathscr{W}_{Q}$ of $K^{\prime}$.

Let $T \subset K$ be the maximal tree that was chosen for the homotopy reading $\mathscr{W}_{0}$. We may insist that $T$ satisfy the following condition, for $q=0$ :

$T_{q} \subset K_{q}$ does not contain any 1-cells that will be removed as a collapse face for any 2-cell $e_{\beta}^{2}$ present in $K_{q}$.

(N.B. Some 1-cells in $T_{q}$ may well be collapse faces for future 2-cells that arise later in $K_{q} \stackrel{n+1}{\wedge} K^{\prime}$.) Note that this condition can be verified by a connectivity argument involving the complex

$$
\left(K_{q}\right)^{1} \cup\left\{e^{2} \in K_{q} \mid e^{2} \text { will be collapsed during } K_{q} \wedge K^{\prime}\right\} .
$$

Suppose that for some $q \geq 0$, we already have a homotopy reading $\mathscr{W}_{q}$ of $K_{q}$ with chosen maximal tree $T_{q} \subset\left(K_{q}\right)^{1}$ such that $W_{q}$ consists of geometric words and $T_{q}$ satisfies $(*)$. By Corollary (3.1.8) and the definition of geometric words, it is clear that an elementary expansion $K_{q} \nearrow_{e, m} K_{q+1}$ or collapse $K_{q} \underset{e, m}{\searrow}$ $K_{q+1}$ of complexes induces an elementary expansion $\mathscr{W}_{q} \nearrow_{e, m} \mathscr{W}_{q+1}$ or collapse $\mathscr{W}_{q} \underset{e, m}{\searrow} \mathscr{W}_{q+1}$ of word systems, where $\mathscr{W}_{q+1}$ is a homotopy reading of $K_{q+1}$ consisting of geometric words. Also, the tree $T_{q+1}$ chosen for the homotopy 
reading $\mathscr{W}_{q+1}$ must satisfy $(*)$, except in one case. That case occurs if a 2 expansion or 3-expansion introduces a new 2-cell $e^{2}$ that has a collapse face $e^{1}$ in $T_{q+1}$.

In that exceptional case, we apply the connectivity argument again to find a maximal tree $\bar{T}_{q+1}$ that satisfies $(*)$. Apply Lemma (2.3.7) to obtain a word system $\overline{\mathscr{W}}_{q+1}$ that is a homotopy reading of $K_{q+1}$ using the maximal tree $\bar{T}_{q+1}$, such that $\mathscr{W}_{q+1}$ is $P_{1}$-equivalent to $\overline{\mathscr{W}}_{q+1}$. Lemma (2.3.7) (and free reduction in the case of 2-words) further asserts that $\overline{\mathscr{W}}_{q+1}$ consists of geometric words, because $\mathscr{W}_{q+1}$ does. Thus, $\mathscr{W}_{q}$ is fully $P_{m}$-equivalent to $\overline{\mathscr{W}}_{q+1}$ where $\overline{\mathscr{W}}_{q+1}$ and $\bar{T}_{q+1}$ satisfy the induction hypothesis.

We conclude that $\mathscr{W}$ is fully $P_{n}$-equivalent to a homotopy reading $\mathscr{W}_{Q}$ of $K^{\prime}$. Since $\mathscr{W}_{Q}$ and $\mathscr{W}^{\prime}$ are two homotopy readings for the same complex, they are fully $P_{n}$-equivalent by Proposition (2.3.8). This establishes $(\mathrm{d}) \Rightarrow(\mathrm{b})$ and completes the proof of the Formal Equivalence Theorem.

\section{APPLICATIONS}

Our proof of Theorem (6.1.1) does not rely on transiency. By this, we mean that an accumulation of top-dimensional words is permitted in the proof of Theorem (4.1.2) $\left(D_{n+1}\right.$-equivalence $\Rightarrow P_{n}$-equivalence). However, an analysis of other parts of the argument establishes the following transiency properties for all dimensions.

Proposition (7.1.1). Suppose that $\mathscr{W}$ and $\overline{\mathscr{W}}$ are word systems of order $n$. If there is a $D_{n+1}$-sequence from $\mathscr{W}$ to $\overline{\mathscr{W}}$ then there is a transient $D_{n+1}$-sequence from $\mathscr{W}$ to $\mathscr{W}$.

Proof. There exists a $P_{n}$-sequence from $\mathscr{W}$ to $\overline{\mathscr{W}}$ by Theorem (6.1.1). Proposition (5.1.6) shows how to convert that $P_{n}$-sequence to a $D_{n+1}$-sequence, which may be assumed transient by Remark (5.1.7).

Proposition (7.1.2). Suppose that $K$ and $\bar{K}$ are $C W$-complexes. If a formal $(n+1)$-deformation $K \stackrel{n+1}{\wedge}$ exists, then there is a transient formal $(n+1)$ deformation from $K$ to $\bar{K}$.

Proof. Let $\mathscr{W}$ and $\overline{\mathscr{W}}$ be homotopy readings of $K$ and $\bar{K}$, respectively. By Proposition (3.3.4), there exist homotopy $n$-deformations $K \stackrel{n}{\wedge} K(\mathscr{W})$ and $\bar{K} \stackrel{n}{\curvearrowleft} K(\overline{\mathscr{W}})$ to model complexes $K(\mathscr{W})$ and $K(\overline{\mathscr{W}})$ of $\mathscr{W}$ and $\overline{\mathscr{W}}$. Thus, there are transient formal $(n+1)$-deformations $K^{n+1} \wedge(\mathscr{W})$ and $\bar{K}^{n+1} \wedge(\overline{\mathscr{W}})$ by Lemma (1.2.3).

Applying Theorem (6.1.1) and Proposition (7.1.1), we conclude that there is a transient $D_{n+1}$-sequence from $\mathscr{W}$ to $\overline{\mathscr{W}}$. We can perform the corresponding sequence of expansions and collapses starting with $K(\mathscr{W})$ to obtain a transient formal $(n+1)$-deformation $K(\mathscr{W})^{n+1} \wedge K^{\prime}$, where $K^{\prime}$ is a model complex for $\overline{\mathscr{W}}$. Proposition (3.3.5) and Lemma (1.2.3) imply that there is a transient formal $(n+1)$-deformation $K^{\prime n+1} \wedge(\overline{\mathscr{W}})$. Thus, we have a transient formal 
$(n+1)$-deformation

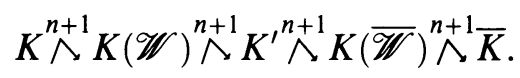

We close with a comment on nonrealizable word systems, such as Example (2.2.3).

Proposition (7.1.3). In a $P_{n}$-equivalence class of word systems, either all of the word systems have geometric realizations or none of them do.

Proof. Suppose $\mathscr{W}$ is $P_{n}$-equivalent to $\mathscr{W}^{\prime}$, and let $K$ be a geometric realization of $\mathscr{W}$. Then there is a model complex $K(\mathscr{W})$ for $\mathscr{W}$, by Proposition (3.3.4). Also, $\mathscr{W}$ is $D_{n+1}$-equivalent to $\mathscr{W}^{\prime}$ by Theorem (4.1.1). Mimicking these $D_{n+1}$-moves as in the proof of Theorem (6.1.1), we obtain a deformation $K(\mathscr{W}) \stackrel{n+1}{\wedge} K\left(\mathscr{W}^{\prime}\right)$ to a model complex for $\mathscr{W}^{\prime}$.

\section{REFERENCES}

1. J. J. Andrews and M. L. Curtis, Free groups and handlebodies, Proc. Amer. Math. Soc. 16 (1965), 192-195.

2. Ronald Brown, On the second relative homotopy group of an adjunction space: An exposition of a theorem of J. H. C. Whitehead, J. London Math. Soc. (2) 22 (1980), 146-152.

3. R. Brown and P. J. Higgins, Colimit theorems for relative homotopy groups, J. Pure Appl. Algebra 22 (1981), 11-41.

4. Robert F. Craggs, Free Heegaard diagrams and extended Nielsen transformations, II, Illinois J. Math. 23 (1979), 101-127.

5. James Dugundji, Topology, Ser. in Adv. Math., Allyn and Bacon, Boston, Mass., 1966.

6. E. Dyer and M.-E. Hamstrom, Completely regular mappings, Fund. Math. 45 (1958), 103118.

7. Wolfgang Metzler, Äquivalenzklassen von Gruppenbeschreibungen, Identitäten und einfacher Homotopietyp in niederen Dimensionen, (C. T. C. Wall, ed.), Homological Group Theory (Proc. Sympos. Durham, 1977), London Math. Soc. Lecture Notes Ser. 36, Cambridge Univ. Press, New York, 1979, pp. 291-326.

8. Reneé Peiffer, Über Identitäten zwischen Relationen, J. Reine Angew. Math. 285 (1976), 17-23.

9. Elvira Strasser Rapaport, Groups of order 1: Some properties of presentations, Acta Math. 121 (1968), 127-150.

10. John G. Ratcliffe, Free and projective crossed modules, J. London Math. Soc. (2) 22 (1980), 66-74.

11. Kurt Reidemeister, Einführung in die Kombinatorische Topologie, Chelsea, New York, 1950.

12. C. T. C. Wall, Formal deformations, Proc. London Math. Soc. (3) 16 (1966), 342-352.

13. George W. Whitehead, Elements of homotopy theory, Graduate Texts in Math., no. 61, Springer-Verlag, New York, 1978.

14. J. H. C. Whitehead, Combinatorial homotopy, II, Bull. Amer. Math. Soc. 55 (1949), 452496.

15. Perrin Wright, Group presentations and formal deformations, Trans. Amer. Math. Soc. 208 (1975), 161-169.

16. Simon F. Young, Contractible 2-complexes, Masters Thesis, Univ. of Cambridge, 1976.

Department of Mathematics, St. Olaf College, Northfield, Minnesota 55057

E-mail address: rab@stolaf.edu 